Electronic Journal of Statistics

Vol. 14 (2020) 1773-1795

ISSN: $1935-7524$

https://doi.org/10.1214/20-EJS1706

\title{
Bias correction in conditional multivariate extremes
}

\author{
Mikael Escobar-Bach ${ }^{\dagger}$, Yuri Goegebeur ${ }^{\ddagger}$ and Armelle Guillou ${ }^{\S}$ \\ † Laboratoire Angevin de Recherche en Mathématiques, UMR 6093 \\ Université d'Angers et CNRS \\ 2 Bd Lavoisier \\ 49045 Angers cedex 01, France \\ e-mail: mikael.escobar-bach@univ-angers.fr \\ $\ddagger$ Department of Mathematics and Computer Science \\ University of Southern Denmark \\ Campusvej 55 \\ 5230 Odense $M$, Denmark \\ e-mail: yuri.goegebeur@imada.sdu.dk \\ $\S$ Institut Recherche Mathématique Avancée, UMR 7501 \\ Université de Strasbourg et CNRS \\ 7 rue René Descartes \\ 67084 Strasbourg cedex, France \\ e-mail: armelle.guillou@math.unistra.fr
}

\begin{abstract}
We consider bias-corrected estimation of the stable tail dependence function in the regression context. To this aim, we first estimate the bias of a smoothed estimator of the stable tail dependence function, and then we subtract it from the estimator. The weak convergence, as a stochastic process, of the resulting asymptotically unbiased estimator of the conditional stable tail dependence function, correctly normalized, is established under mild assumptions, the covariate argument being fixed. The finite sample behaviour of our asymptotically unbiased estimator is then illustrated on a simulation study and compared to two alternatives, which are not bias corrected. Finally, our methodology is applied to a dataset of air pollution measurements.
\end{abstract}

MSC 2010 subject classifications: Primary 62G32, 62G05, 62G20; secondary 60F05, 60G70.

Keywords and phrases: Bias correction, conditional stable tail dependence function, stochastic convergence.

Received March 2019.

\section{Contents}

1 Introduction . . . . . . . . . . . . . . . . . . . . 1774

*This work was supported by a research grant (VKR023480) from VILLUM FONDEN and the research of Armelle Guillou was also supported by the French National Research Agency under the grant ANR-19-CE40-0013-01/ExtremReg project and an International Emerging Action (IEA-00179). Computation/simulation for the work described in this paper was supported by the DeIC National HPC Centre, SDU. The authors sincerely thank the Editor and Associate Editor for their helpful comments and suggestions that led to substantial improvement of the paper. 
2 Estimators and convergence results . . . . . . . . . . . . . . 1775

2.1 Asymptotic result for $\widehat{L}_{k}\left(\cdot \mid x_{0}\right)$ under a third order condition . . . 1778

2.2 Smoothed estimator for $L\left(\cdot \mid x_{0}\right) \ldots \ldots \ldots \ldots \ldots$

2.3 Estimation of $\rho\left(x_{0}\right)$ and $\alpha\left(n / k \mid x_{0}\right) M\left(y \mid x_{0}\right) \ldots \ldots \ldots \ldots$

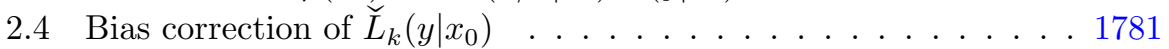

3 Simulation study . . . . . . . . . . . . . . . . . . . . . 1783

4 Application to air pollution data . . . . . . . . . . . 1787

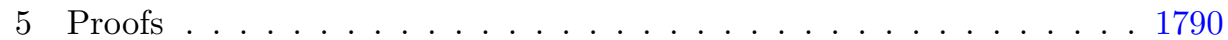

5.1 Proof of Theorem $2.1 \ldots \ldots \ldots \ldots$. . . . . . . . . 1790

5.2 Proof of Theorem $2.2 \ldots \ldots \ldots \ldots$

5.3 Proof of Theorem 2.3 . . . . . . . . . . . . . . . 1792

5.4 Proof of Theorem 2.4 . . . . . . . . . . . . . . . 1793

5.5 Proof of Theorem 2.5 . . . . . . . . . . . . . . . 1793

References . . . . . . . . . . . . . . . . . . . . 1794

\section{Introduction}

Most of the practical problems involving extreme events are inherently multivariate. Consequently, being able to estimate the extremal dependence between random variables is useful. To this aim, we can either use some extremal coefficients, that give a representative picture of the full dependency structure (see, e.g., Ledford and Tawn, 1997), or functions, such as the spectral distribution function or the stable tail dependence function, that provide a full characterization of the extreme dependence between variables. We refer to Beirlant et al. (2004) and de Haan and Ferreira (2006), and the references therein, for more details. In this paper, we will focus on the stable tail dependence function, which can be defined as follows.

For any arbitrary dimension $d$, let $\left(Y^{(1)}, \ldots, Y^{(d)}\right)$ be a random vector with continuous marginal distribution functions $F_{1}, \ldots, F_{d}$. The stable tail dependence function is defined for each $y_{i} \in \mathbb{R}_{+}, i=1, \ldots, d$, as

$$
\lim _{t \rightarrow \infty} t \mathbb{P}\left(1-F_{1}\left(Y^{(1)}\right) \leqslant t^{-1} y_{1} \text { or } \ldots \text { or } 1-F_{d}\left(Y^{(d)}\right) \leqslant t^{-1} y_{d}\right)=L\left(y_{1}, \ldots, y_{d}\right),
$$

provided that this limit exists. We refer to Huang (1992), and de Haan and Ferreira (2006) for more details.

Several estimators for $L$ have been proposed in the literature, see, e.g., Huang (1992), Drees and Huang (1998), Fils-Villetard et al. (2008), Bücher et al. (2014), but as usual in the extreme value framework, the classical estimators are affected by bias, which often complicates their practical application. To solve this issue, Fougères et al. (2015) and Beirlant et al. (2016) have introduced bias-corrected estimators and they have established the main properties of their estimators as stochastic processes.

Taking care of the bias is important, but in practical applications, we are also often faced with the presence of covariates in addition to the random vector $\left(Y^{(1)}, \ldots, Y^{(d)}\right)$. It is thus important to be able to estimate the stable tail dependence function when random covariates $X$ are present, i.e., to consider 
the regression situation with a multivariate response. In that case, we want to describe the extremal dependence between the variables $\left(Y^{(1)}, \ldots, Y^{(d)}\right)$ given some observed value $x$ for the covariate $X \in \mathbb{R}^{p}$. Thus, the notion of conditional stable tail dependence function $L(\cdot \mid x)$ can be introduced and the classical framework (1.1) can be extended into

$$
\begin{gathered}
\lim _{t \rightarrow \infty} t \mathbb{P}\left(1-F_{1}\left(Y^{(1)} \mid X\right) \leqslant t^{-1} y_{1} \text { or } \ldots \text { or } 1-F_{d}\left(Y^{(d)} \mid X\right) \leqslant t^{-1} y_{d} \mid X=x\right) \\
=L\left(y_{1}, \ldots, y_{d} \mid x\right),
\end{gathered}
$$

where $F_{j}(\cdot \mid x), j=1, \ldots, d$, denote the continuous conditional distribution function of $Y^{(j)}$ given $X=x$. To the best of our knowledge, the estimation of the conditional stable tail dependence function has only been studied very recently by Escobar-Bach et al. (2018b), where a local estimator was proposed and its weak convergence as a stochastic process was established. In related work, Gardes and Girard (2015) introduced an estimator for the conditional tail copula and studied its finite dimensional convergence. However, being in the regression context, of course does not solve the bias problem of the estimator of $L(\cdot \mid x)$. Thus, combining bias-correction and regression will be the subject of this paper. As far as we know, this topic is completely new in the literature.

The remainder of the paper is organized as follows. In Section 2, we introduce our bias-corrected estimator of the conditional stable tail dependence function and we establish its weak convergence as a stochastic process, the covariate being fixed. Then in Section 3, we illustrate the performance of this estimator on a small simulation study where we compare it with two alternatives, that are not asymptotically unbiased. Section 4 is devoted to a data analysis of air pollution measurements. All the proofs are postponed to Section 5 .

\section{Estimators and convergence results}

Denote $(Y, X):=\left(Y^{(1)}, \ldots, Y^{(d)}, X\right)$, a random vector satisfying (1.2), and let $\left(Y_{1}, X_{1}\right), \ldots,\left(Y_{n}, X_{n}\right)$, be independent copies of $(Y, X)$, where $X$ has density function $f$. We introduce a local estimator for $L$, based on an empirical version of the left-hand side of (1.2), for large values of $t$. As is usual in the extreme value context, we consider an intermediate sequence $k=k_{n}$, i.e., $k \rightarrow \infty$ as $n \rightarrow \infty$ with $k / n \rightarrow 0$. Since the margins $F_{j}(\cdot \mid x)$ appearing in (1.2) are unknown in practice, we have to replace them by estimators such as the empirical kernel estimator

$$
\widehat{F}_{n, j}(y \mid x):=\frac{\sum_{i=1}^{n} K_{c}\left(x-X_{i}\right) \mathbb{1}_{\left\{Y_{i}^{(j)} \leqslant y\right\}}}{\sum_{i=1}^{n} K_{c}\left(x-X_{i}\right)}, j=1, \ldots, d,
$$

where $K_{c}(\cdot):=K(\cdot / c) / c^{p}$ with $K$ a density function on $\mathbb{R}^{p}$, and $c:=c_{n}$ is a positive non-random sequence satisfying $c_{n} \rightarrow 0$ as $n \rightarrow \infty$. Denote with $y:=\left(y_{1}, \ldots, y_{d}\right)$ a vector of the positive quadrant $\mathbb{R}_{+}^{d}$. According to Escobar- 
Bach et al. (2018b)

$\widehat{L}_{k}(y \mid x):=\frac{\frac{1}{k} \sum_{i=1}^{n} K_{h}\left(x-X_{i}\right) \mathbb{1}_{\left\{1-\hat{F}_{n, 1}\left(Y_{i}^{(1)} \mid X_{i}\right) \leqslant \frac{k}{n} y_{1} \text { or } \ldots \text { or } 1-\hat{F}_{n, d}\left(Y_{i}^{(d)} \mid X_{i}\right) \leqslant \frac{k}{n} y_{d}\right\}}}{\frac{1}{n} \sum_{i=1}^{n} K_{h}\left(x-X_{i}\right)}$

with $h:=h_{n}$ a positive non-random sequence satisfying $h_{n} \rightarrow 0$ as $n \rightarrow \infty$, is an estimator of the conditional stable tail dependence function $L(y \mid x)$. Note that in $\widehat{F}_{n, j}(y \mid x)$ and $\widehat{L}_{k}(y \mid x)$ the same kernel function $K$ has been used, but they can of course be taken different. As in Escobar-Bach et al. (2018b), the bandwidths for $\widehat{F}_{n, j}$ and $\widehat{L}_{k}$ are though different. This non-parametric estimator of $L(\cdot \mid x)$ is in construction similar to the estimator for the conditional copula function introduced in Veraverbeke et al. (2011). The purpose of the two papers is different though. In our paper we study the asymptotic properties of an estimator of extreme tail dependence, while in Veraverbeke et al. (2011), focus was on the asymptotic behavior of an estimator for the conditional copula function.

The aim of the paper is to propose an asymptotically unbiased estimator for $L(\cdot \mid x)$. To the best of our knowledge this topic has not been considered previously in the literature in the regression context, in contrast to the classical framework without covariates where we can mention the contributions of Fougères et al. (2015) and Beirlant et al. (2016).

The main results of the paper will be derived as stochastic weak convergence results for processes in $y \in[\varepsilon, T]^{d}$, for any $\varepsilon>0$ and $T>\varepsilon$, but with the covariate argument fixed, meaning that we will focus our study only around one reference position $x_{0} \in \operatorname{Int}\left(S_{X}\right)$, the interior of the support $S_{X}$ of $f$, assumed to be non-empty. To this aim, we need to introduce some conditions mentioned below and well-known in the extreme value framework. Let $\|$.$\| be some norm$ on $\mathbb{R}^{p}$, and denote by $B_{x}(\tau)$ the closed ball with respect to $\|\cdot\|$, centered at $x$ and with radius $\tau>0$. The event $A_{t, y}$ is defined for any $t>0$ and $y \in \mathbb{R}_{+}^{d}$ as

$$
A_{t, y}:=\left\{1-F_{1}\left(Y^{(1)} \mid X\right) \leqslant t^{-1} y_{1} \text { or } \ldots \text { or } 1-F_{d}\left(Y^{(d)} \mid X\right) \leqslant t^{-1} y_{d}\right\} .
$$

First order condition: The limit in (1.2) exists for all $x \in S_{X}$ and $y \in \mathbb{R}_{+}^{d}$, and the convergence is uniform on $[0, T]^{d} \times B_{x_{0}}(\tau)$ for any $T>0$ and $a \tau>0$.

Second order condition: For any $x \in S_{X}$ there exist a positive function $\alpha(\cdot \mid x)$ such that $\alpha(t \mid x) \rightarrow 0$ as $t \rightarrow \infty$ and a non null function $M(\cdot \mid x)$ such that for all $y \in \mathbb{R}_{+}^{d}$

$$
\lim _{t \rightarrow \infty} \frac{1}{\alpha(t \mid x)}\left\{t \mathbb{P}\left(A_{t, y} \mid X=x\right)-L(y \mid x)\right\}=M(y \mid x),
$$

uniformly on $[0, T]^{d} \times B_{x_{0}}(\tau)$ for any $T>0$ and $a \tau>0$.

Third order condition: For any $x \in S_{X}$ there exist a positive function $\beta(\cdot \mid x)$ such that $\beta(t \mid x) \rightarrow 0$ as $t \rightarrow \infty$ and a non null function $N(\cdot \mid x)$ such that for all 
$y \in \mathbb{R}_{+}^{d}$

$$
\lim _{t \rightarrow \infty} \frac{1}{\beta(t \mid x)}\left\{\frac{t \mathbb{P}\left(A_{t, y} \mid X=x\right)-L(y \mid x)}{\alpha(t \mid x)}-M(y \mid x)\right\}=N(y \mid x),
$$

uniformly on $[0, T]^{d} \times B_{x_{0}}(\tau)$ for any $T>0$ and a $\tau>0$, and where $N$ is not a multiple of $M$.

Note that these assumptions imply that the functions $\alpha(\cdot \mid x)$ and $\beta(\cdot \mid x)$ are both regularly varying with indices $\rho(x)$ and $\rho^{\prime}(x)$ respectively which are non positive. In the sequel we assume that both indices are negative. Remark also that the functions $L(\cdot \mid x), M(\cdot \mid x)$ and $N(\cdot \mid x)$ have a homogeneity property, that is $L(a y \mid x)=a L(y \mid x), M(a y \mid x)=a^{1-\rho(x)} M(y \mid x), N(a y \mid x)=a^{1-\rho(x)-\rho^{\prime}(x)} N(y \mid x)$ for all $a>0$ and all $y \in \mathbb{R}_{+}^{d}$.

Due to the regression context, we need some Hölder-type conditions.

Assumption $\left(\mathcal{F}_{m}\right)$. There exist $M_{F_{j}}>0$ and $\eta_{F_{j}}>0$ such that $\mid F_{j}(y \mid x)-$ $F_{j}(y \mid z) \mid \leqslant M_{F_{j}}\|x-z\|^{\eta_{F_{j}}}$, for all $y \in \mathbb{R}$, all $(x, z) \in S_{X} \times S_{X}$ and $j=1, \ldots, d$.

Assumption $(\mathcal{D})$. There exist $M_{f}>0$ and $\eta_{f}>0$ such that $|f(x)-f(z)| \leqslant$ $M_{f}\|x-z\|^{\eta_{f}}$, for all $(x, z) \in S_{X} \times S_{X}$.

Assumption $(\mathcal{L})$. There exist $M_{L}>0$ and $\eta_{L}>0$ such that $|L(y \mid x)-L(y \mid z)| \leqslant$ $M_{L}\|x-z\|^{\eta_{L}}$, for all $(x, z) \in B_{x_{0}}(\tau) \times B_{x_{0}}(\tau), \tau>0$, and $y \in[0, T]^{d}, T>0$.

Assumption $(\mathcal{A})$. There exist $M_{\alpha}>0$ and $\eta_{\alpha}>0$ such that $|\alpha(t \mid x)-\alpha(t \mid z)| \leqslant$ $M_{\alpha}\|x-z\|^{\eta_{\alpha}}$, for all $(x, z) \in S_{X} \times S_{X}$ and $t \geqslant 0$.

Assumption $(\mathcal{B})$. There exist $M_{\beta}>0$ and $\eta_{\beta}>0$ such that $|\beta(t \mid x)-\beta(t \mid z)| \leqslant$ $M_{\beta}\|x-z\|^{\eta_{\beta}}$, for all $(x, z) \in S_{X} \times S_{X}$ and $t \geqslant 0$.

Assumption $(\mathcal{M})$. There exist $M>0$ and $\eta_{M}>0$ such that $\mid M(y \mid x)-$ $M(y \mid z) \mid \leqslant M\|x-z\|^{\eta_{M}}$, for all $(x, z) \in B_{x_{0}}(\tau) \times B_{x_{0}}(\tau), \tau>0$, and $y \in$ $[0, T]^{d}, T>0$.

Also a condition is required on the kernel function $K$.

Assumption $(\mathcal{K}) . K$ is a bounded density function on $\mathbb{R}^{p}$ with support $S_{K}$ included in the unit ball of $\mathbb{R}^{p}$ with respect to the norm $\|$.$\| . Moreover, we assume$ that there exists $\delta, m>0$ such that $B_{0}(\delta) \subset S_{K}$ and $K(u) \geqslant m$ for all $u \in$ $B_{0}(\delta)$, and $K$ belongs to the linear span (the set of finite linear combinations) of functions $k \geqslant 0$ satisfying the following property: the subgraph of $k,\{(s, u)$ : $k(s) \geqslant u\}$, can be represented as a finite number of Boolean operations among sets of the form $\{(s, u): q(s, u) \geqslant \varphi(u)\}$, where $q$ is a polynomial on $\mathbb{R}^{p} \times \mathbb{R}$ and $\varphi$ is an arbitrary real function.

The latter assumption is common, and used already in, e.g., Giné and Guillou (2002) and Escobar-Bach et al. (2018a,b), and allows to measure the discrepancy 
between the conditional distribution function $F_{j}(\cdot \mid x)$ and its empirical kernel version $\widehat{F}_{n, j}(\cdot \mid x)$.

\subsection{Asymptotic result for $\widehat{L}_{k}\left(\cdot \mid x_{0}\right)$ under a third order condition}

Escobar-Bach et al. (2018b) have established the weak convergence of $\widehat{L}_{k}\left(y \mid x_{0}\right)$ as a stochastic process in $y \in[0, T]^{d}$ and for a fixed covariate position $x_{0} \in$ $\mathbb{R}^{p}$, under the second order condition. In order to construct an asymptotically unbiased estimator for $L\left(\cdot \mid x_{0}\right)$, the third order condition is required and thus we need to know if under this new condition, a similar convergence result can be stated.

For any $T>0$, let $D\left([0, T]^{d}\right)$ be the space of functions on $[0, T]^{d}$ that are right-continuous and have left-hand limits. All our weak convergence results will be derived in the Skorohod space $D\left([0, T]^{d}\right)$ equipped with the sup norm.

Theorem 2.1. Assume the third order condition, $y \rightarrow M(y \mid x)$ continuous on $[0, T]^{d}$ and $(x, y) \rightarrow N(y \mid x)$ continuous on $B_{x_{0}}(\tau) \times[0, T]^{d}$, with $B_{x_{0}}(\tau) \subset S_{X}$. Also suppose that there exists $b>0$ with $f(x) \geqslant b, \forall x \in S_{X} \subset \mathbb{R}^{p}$ and $f$ bounded. Under $\left(\mathcal{F}_{m}\right),(\mathcal{D}),(\mathcal{L}),(\mathcal{A}),(\mathcal{B}),(\mathcal{M}),(\mathcal{K})$, and assuming that there exists an $\varepsilon>0$ such that for $n$ sufficiently large

$$
\inf _{x \in S_{X}} \lambda\left(\left\{u \in B_{0}(1): x-c u \in S_{X}\right\}\right)>\varepsilon,
$$

where $\lambda$ denotes the Lebesgue measure, consider sequences $k \rightarrow \infty, h \rightarrow 0$ and $c \rightarrow 0$ as $n \rightarrow \infty$ such that $k / n \rightarrow 0$ with

$$
\begin{aligned}
\sqrt{k h^{p}} h^{\min \left(\eta_{f}, \eta_{L}, \eta_{\alpha}\right)} & \longrightarrow 0, \\
\sqrt{k h^{p}} \alpha\left(n / k \mid x_{0}\right) h^{\min \left(\eta_{M}, \eta_{\beta}\right)} & \longrightarrow 0, \\
\sqrt{k h^{p}} \alpha\left(n / k \mid x_{0}\right) & \longrightarrow \infty \\
\sqrt{k h^{p}} \alpha\left(n / k \mid x_{0}\right) \beta\left(n / k \mid x_{0}\right) & \longrightarrow \mu_{1}\left(x_{0}\right) \in \mathbb{R}_{+},
\end{aligned}
$$

and for some $q>1$ and $0<\eta<\min \left(\eta_{F_{1}}, \ldots, \eta_{F_{d}}\right)$

$$
n \sqrt{\frac{h^{p}}{k}} \max \left(\sqrt{\frac{|\log c|^{q}}{n c^{p}}}, c^{\eta}\right) \longrightarrow 0 .
$$

Then the process

$$
\begin{array}{r}
\left\{\sqrt { k h ^ { p } } \left(\widehat{L}_{k}\left(y \mid x_{0}\right)-L\left(y \mid x_{0}\right)-\alpha\left(\frac{n}{k} \mid x_{0}\right) M\left(y \mid x_{0}\right)\right.\right. \\
\left.\left.-\alpha\left(\frac{n}{k} \mid x_{0}\right) \beta\left(\frac{n}{k} \mid x_{0}\right) N\left(y \mid x_{0}\right)\right), y \in[0, T]^{d}\right\}
\end{array}
$$

weakly converges in $D\left([0, T]^{d}\right)$ towards a tight centered Gaussian process $\{B(y)$, $\left.y \in[0, T]^{d}\right\}$, for any $T>0$, with covariance structure given by

$$
\operatorname{Cov}\left(B(y), B\left(y^{\prime}\right)\right)=\frac{\|K\|_{2}^{2}}{f\left(x_{0}\right)}\left(L\left(y \mid x_{0}\right)+L\left(y^{\prime} \mid x_{0}\right)-L\left(y \vee y^{\prime} \mid x_{0}\right)\right),
$$


where $y, y^{\prime} \in[0, T]^{d}$ and $\|K\|_{2}:=\sqrt{\int_{S_{K}} K^{2}(u) d u}$.

\subsection{Smoothed estimator for $L\left(\cdot \mid x_{0}\right)$}

Inspired by the homogeneity of $L\left(\cdot \mid x_{0}\right)$, consider now the rescaled statistic

$$
\widehat{L}_{k, a}\left(y \mid x_{0}\right):=\frac{1}{a} \widehat{L}_{k}\left(a y \mid x_{0}\right)
$$

for a positive scale parameter $a$. Our uncorrected (in terms of bias) estimator for $L\left(\cdot \mid x_{0}\right)$ will be the following weighted version of the rescaled statistic, defined for any $r>1$ and $\xi>0$ as

$$
\breve{L}_{k}\left(y \mid x_{0}\right):=\left(\frac{\int_{1}^{r} \widehat{L}_{k, a}^{\xi}\left(y \mid x_{0}\right) d a}{r-1}\right)^{1 / \xi} .
$$

The weak convergence of this new estimator as a stochastic process is established in the following theorem.

Theorem 2.2. Under the assumptions of Theorem 2.1 together with

$$
\sqrt{k h^{p}} \alpha^{2}\left(n / k \mid x_{0}\right) \rightarrow \mu_{2}\left(x_{0}\right) \in \mathbb{R}_{+}
$$

for any $r>1$ and $\xi>0$, we have

$$
\begin{aligned}
& \sqrt{k h^{p}}\left\{\breve{L}_{k}\left(y \mid x_{0}\right)-L\left(y \mid x_{0}\right)-\alpha\left(\frac{n}{k} \mid x_{0}\right) M\left(y \mid x_{0}\right) c\left(r ; \rho\left(x_{0}\right)\right)\right. \\
& \left.-\alpha\left(\frac{n}{k} \mid x_{0}\right) \beta\left(\frac{n}{k} \mid x_{0}\right) N\left(y \mid x_{0}\right) c\left(r ; \rho\left(x_{0}\right)+\rho^{\prime}\left(x_{0}\right)\right)-\frac{\alpha^{2}\left(n / k \mid x_{0}\right)}{2} \frac{M^{2}\left(y \mid x_{0}\right)}{L\left(y \mid x_{0}\right)} d\left(r, \xi ; \rho\left(x_{0}\right)\right)\right\} \\
& \quad \stackrel{d}{\longrightarrow} \frac{1}{r-1} \int_{1}^{r} \frac{B(a y)}{a} d a,
\end{aligned}
$$

in $D\left([\varepsilon, T]^{d}\right)$, for every $\varepsilon>0$ and $T>\varepsilon$, where $B$ is defined in Theorem 2.1 and

$$
\begin{aligned}
c\left(r ; \rho\left(x_{0}\right)\right) & :=\frac{r^{1-\rho\left(x_{0}\right)}-1}{(r-1)\left(1-\rho\left(x_{0}\right)\right)}, \\
d\left(r, \xi ; \rho\left(x_{0}\right)\right) & :=\left[c\left(r ; 2 \rho\left(x_{0}\right)\right)-c^{2}\left(r ; \rho\left(x_{0}\right)\right)\right](\xi-1) .
\end{aligned}
$$

Based on this result, in order to construct an asymptotically unbiased estimator for $L\left(\cdot \mid x_{0}\right)$, we need now to estimate $\rho\left(x_{0}\right)$ and $\alpha_{k}\left(y \mid x_{0}\right):=\alpha\left(n / k \mid x_{0}\right) M\left(y \mid x_{0}\right)$. This is the aim of the next section.

\subsection{Estimation of $\rho\left(x_{0}\right)$ and $\alpha\left(n / k \mid x_{0}\right) M\left(y \mid x_{0}\right)$}

Let $\left(\xi_{1}, \xi_{2}, \xi_{3}, \xi_{4}\right) \in \mathbb{R}_{+}^{4}, r_{1} \neq r_{2}>1$ and $s>0$. Now, define

$$
I(y ; r, t ; s):=\frac{1}{r-1} \int_{1}^{r} \frac{B(a s y)}{a} d a-\frac{1}{t-1} \int_{1}^{t} \frac{B(a s y)}{a} d a .
$$


We propose to estimate $\rho\left(x_{0}\right)$ by

$$
\breve{\rho}_{k}\left(x_{0}\right):=1-\frac{1}{\log s} \log \left(\frac{\left(\frac{\int_{1}^{r_{1}} \widehat{L}_{k, a}^{\xi_{1}}\left(s y \mid x_{0}\right) d a}{r_{1}-1}\right)^{1 / \xi_{1}}-\left(\frac{\int_{1}^{r_{2}} \hat{L}_{k, a}^{\xi_{2}}\left(s y \mid x_{0}\right) d a}{r_{2}-1}\right)^{1 / \xi_{2}}}{\left(\frac{\int_{1}^{r_{1}} \widehat{L}_{k, a}^{\xi_{3}}\left(y \mid x_{0}\right) d a}{r_{1}-1}\right)^{1 / \xi_{3}}-\left(\frac{\int_{1}^{r_{2}} \hat{L}_{k, a}^{\xi_{4}}\left(y \mid x_{0}\right) d a}{r_{2}-1}\right)^{1 / \xi_{4}}}\right) .
$$

Theorem 2.3. Under the assumptions of Theorem 2.1, and additionally assuming that $M$ never vanishes except on the axes and that $\sqrt{k h^{p}} \alpha^{2}\left(n / k \mid x_{0}\right) \rightarrow$ $\mu_{2}\left(x_{0}\right) \in \mathbb{R}_{+}$, for any $\left(\xi_{1}, \xi_{2}, \xi_{3}, \xi_{4}\right) \in \mathbb{R}_{+}^{4}, r_{1} \neq r_{2}>1$ and $s>0$, we have

$$
\begin{aligned}
& \sqrt{k h^{p}} \alpha\left(\frac{n}{k} \mid x_{0}\right)\left\{\check{\rho}_{k}\left(x_{0}\right)-\rho\left(x_{0}\right)+\frac{\alpha\left(n / k \mid x_{0}\right)}{2 \log s} \frac{M\left(y \mid x_{0}\right)}{L\left(y \mid x_{0}\right)}\right. \\
& \times\left[s^{-\rho\left(x_{0}\right)} \frac{d\left(r_{1}, \xi_{1} ; \rho\left(x_{0}\right)\right)-d\left(r_{2}, \xi_{2} ; \rho\left(x_{0}\right)\right)}{c\left(r_{1} ; \rho\left(x_{0}\right)\right)-c\left(r_{2} ; \rho\left(x_{0}\right)\right)}-\frac{d\left(r_{1}, \xi_{3} ; \rho\left(x_{0}\right)\right)-d\left(r_{2}, \xi_{4} ; \rho\left(x_{0}\right)\right)}{c\left(r_{1} ; \rho\left(x_{0}\right)\right)-c\left(r_{2} ; \rho\left(x_{0}\right)\right)}\right] \\
& \left.+\beta\left(\frac{n}{k} \mid x_{0}\right) \frac{N\left(y \mid x_{0}\right)}{M\left(y \mid x_{0}\right)} \frac{s^{-\rho^{\prime}\left(x_{0}\right)}-1}{\log s} \frac{c\left(r_{1} ; \rho\left(x_{0}\right)+\rho^{\prime}\left(x_{0}\right)\right)-c\left(r_{2} ; \rho\left(x_{0}\right)+\rho^{\prime}\left(x_{0}\right)\right)}{c\left(r_{1} ; \rho\left(x_{0}\right)\right)-c\left(r_{2} ; \rho\left(x_{0}\right)\right)}\right\} \\
& \stackrel{d}{\longrightarrow}-\frac{\frac{s^{\rho\left(x_{0}\right)-1}}{\log s} I\left(y ; r_{1}, r_{2} ; s\right)-\frac{1}{\log s} I\left(y ; r_{1}, r_{2} ; 1\right)}{M\left(y \mid x_{0}\right)\left[c\left(r_{1} ; \rho\left(x_{0}\right)\right)-c\left(r_{2} ; \rho\left(x_{0}\right)\right)\right]}
\end{aligned}
$$

in $D\left([\varepsilon, T]^{d}\right)$, for every $\varepsilon>0$ and $T>\varepsilon$, where $B$ is defined in Theorem 2.1.

Let $\left(\xi_{5}, \xi_{6}\right) \in \mathbb{R}_{+}^{2}$ and $r_{3} \neq r_{4}>1$. To estimate $\alpha_{k}\left(y \mid x_{0}\right)$, we propose

$$
\check{\alpha}_{k}\left(y \mid x_{0}\right):=\frac{\left(\frac{\int_{1}^{r_{3}} \widehat{L}_{k, a}^{\xi_{5}}\left(y \mid x_{0}\right) d a}{r_{3}-1}\right)^{1 / \xi_{5}}-\left(\frac{\int_{1}^{r_{4}} \widehat{L}_{k, a}^{\xi_{6}}\left(y \mid x_{0}\right) d a}{r_{4}-1}\right)^{1 / \xi_{6}}}{c\left(r_{3} ; \check{\rho}_{k}\left(x_{0}\right)\right)-c\left(r_{4} ; \check{\rho}_{k}\left(x_{0}\right)\right)} .
$$

In the sequel, we denote by $c^{\prime}\left(r ; \rho\left(x_{0}\right)\right)$ the derivative of $c\left(r ; \rho\left(x_{0}\right)\right)$ with respect to $\rho\left(x_{0}\right)$, and we use the following notations:

$$
\begin{aligned}
& \widetilde{M}\left(y \mid x_{0}\right):= \\
& \frac{M\left(y \mid x_{0}\right)}{2 L\left(y \mid x_{0}\right)}\left(\frac{d\left(r_{3}, \xi_{5} ; \rho\left(x_{0}\right)\right)-d\left(r_{4}, \xi_{6} ; \rho\left(x_{0}\right)\right)}{c\left(r_{3} ; \rho\left(x_{0}\right)\right)-c\left(r_{4} ; \rho\left(x_{0}\right)\right)}+\left[c^{\prime}\left(r_{3} ; \rho\left(x_{0}\right)\right)-c^{\prime}\left(r_{4} ; \rho\left(x_{0}\right)\right)\right]\right. \\
& \left.\times\left[\frac{s^{-\rho\left(x_{0}\right)}\left[d\left(r_{1}, \xi_{1} ; \rho\left(x_{0}\right)\right)-d\left(r_{2}, \xi_{2} ; \rho\left(x_{0}\right)\right)\right]-\left[d\left(r_{1}, \xi_{3} ; \rho\left(x_{0}\right)\right)-d\left(r_{2}, \xi_{4} ; \rho\left(x_{0}\right)\right)\right]}{\log s\left[c\left(r_{1} ; \rho\left(x_{0}\right)\right)-c\left(r_{2} ; \rho\left(x_{0}\right)\right)\right]\left[c\left(r_{3} ; \rho\left(x_{0}\right)\right)-c\left(r_{4} ; \rho\left(x_{0}\right)\right)\right]}\right]\right) \\
& \widetilde{N}\left(y \mid x_{0}\right):= \\
& \frac{N\left(y \mid x_{0}\right)}{M\left(y \mid x_{0}\right)}\left(\frac{c\left(r_{3} ; \rho\left(x_{0}\right)+\rho^{\prime}\left(x_{0}\right)\right)-c\left(r_{4} ; \rho\left(x_{0}\right)+\rho^{\prime}\left(x_{0}\right)\right)}{c\left(r_{3} ; \rho\left(x_{0}\right)\right)-c\left(r_{4} ; \rho\left(x_{0}\right)\right)}\right. \\
& +\frac{\left[c^{\prime}\left(r_{3} ; \rho\left(x_{0}\right)\right)-c^{\prime}\left(r_{4} ; \rho\left(x_{0}\right)\right)\right]\left[c\left(r_{1} ; \rho\left(x_{0}\right)+\rho^{\prime}\left(x_{0}\right)\right)-c\left(r_{2} ; \rho\left(x_{0}\right)+\rho^{\prime}\left(x_{0}\right)\right)\right]}{\left[c\left(r_{1} ; \rho\left(x_{0}\right)\right)-c\left(r_{2} ; \rho\left(x_{0}\right)\right)\right]\left[c\left(r_{3} ; \rho\left(x_{0}\right)\right)-c\left(r_{4} ; \rho\left(x_{0}\right)\right)\right]} \\
& \left.\times \frac{s^{\rho^{\prime}\left(x_{0}\right)}-1}{\log s}\right) .
\end{aligned}
$$


Theorem 2.4. Under the assumptions of Theorem 2.3, we have

$$
\begin{aligned}
\sqrt{k h^{p}} & \alpha\left(\frac{n}{k} \mid x_{0}\right)\left\{\frac{\check{\alpha}_{k}\left(y \mid x_{0}\right)}{\alpha\left(n / k \mid x_{0}\right) M\left(y \mid x_{0}\right)}-1-\alpha\left(\frac{n}{k} \mid x_{0}\right) \widetilde{M}\left(y \mid x_{0}\right)\right. \\
& \left.-\beta\left(\frac{n}{k} \mid x_{0}\right) \tilde{N}\left(y \mid x_{0}\right)\right\} \stackrel{d}{\longrightarrow} \frac{1}{c\left(r_{3} ; \rho\left(x_{0}\right)\right)-c\left(r_{4} ; \rho\left(x_{0}\right)\right)} \frac{1}{M\left(y \mid x_{0}\right)}\left\{I\left(y ; r_{3}, r_{4} ; 1\right)\right. \\
& \left.-\frac{c^{\prime}\left(r_{4} ; \rho\left(x_{0}\right)\right)-c^{\prime}\left(r_{3} ; \rho\left(x_{0}\right)\right)}{c\left(r_{1} ; \rho\left(x_{0}\right)\right)-c\left(r_{2} ; \rho\left(x_{0}\right)\right)}\left[\frac{s^{\rho\left(x_{0}\right)-1}}{\log s} I\left(y ; r_{1}, r_{2} ; s\right)-\frac{1}{\log s} I\left(y ; r_{1}, r_{2} ; 1\right)\right]\right\}
\end{aligned}
$$

in $D\left([\varepsilon, T]^{d}\right)$, for every $\varepsilon>0$ and $T>\varepsilon$, where $B$ is defined in Theorem 2.1.

\subsection{Bias correction of $\breve{L}_{k}\left(y \mid x_{0}\right)$}

Now we have all the ingredients to construct an asymptotically unbiased estimator for $L\left(\cdot \mid x_{0}\right)$ by removing from $\breve{L}_{k}\left(y \mid x_{0}\right)$ the bias term where $\alpha\left(n / k \mid x_{0}\right) M\left(y \mid x_{0}\right)$ together with the second order rate parameter $\rho\left(x_{0}\right)$ have been estimated externally, using the same intermediate sequence $\bar{k}=\bar{k}_{n}$, which is such that $k=o(\bar{k})$. This idea has been originally proposed by Gomes and co-authors (see, e.g., Gomes et al., 2008; Caeiro et al., 2009) in the univariate framework and has the advantage that the variance of the bias-corrected estimator and the uncorrected one is the same. Thus, we propose the following bias-corrected estimator for $L\left(\cdot \mid x_{0}\right)$

$$
\bar{L}_{k, \bar{k}}\left(y \mid x_{0}\right):=\breve{L}_{k}\left(y \mid x_{0}\right)-\check{\alpha}_{\bar{k}}\left(y \mid x_{0}\right) c\left(r ; \check{\rho}_{\bar{k}}\left(x_{0}\right)\right)\left(\frac{\bar{k}}{k}\right)^{\check{\rho}_{\bar{k}}\left(x_{0}\right)} .
$$

Theorem 2.5. Assume the third order condition, $M$ never vanishes except on the axes, $y \rightarrow M(y \mid x)$ continuous on $[0, T]^{d}$ and $(x, y) \rightarrow N(y \mid x)$ continuous on $B_{x_{0}}(\tau) \times[0, T]^{d}$, with $B_{x_{0}}(\tau) \subset S_{X}$. Also suppose that there exists $b>0$ with $f(x) \geqslant b, \forall x \in S_{X} \subset \mathbb{R}^{p}$ and $f$ bounded. Under $\left(\mathcal{F}_{m}\right),(\mathcal{D}),(\mathcal{L}),(\mathcal{A}),(\mathcal{B}),(\mathcal{M})$, $(\mathcal{K})$, and assuming that there exists an $\varepsilon>0$ such that for $n$ sufficiently large

$$
\inf _{x \in S_{X}} \lambda\left(\left\{u \in B_{0}(1): x-c u \in S_{X}\right\}\right)>\varepsilon
$$

consider sequences $k \rightarrow \infty, h \rightarrow 0, c \rightarrow 0$ as $n \rightarrow \infty$ and $\bar{k}$ such that $k=o(\bar{k})$, $\bar{k} / n \rightarrow 0$, and with

$$
\begin{aligned}
\sqrt{\bar{k} h^{p}} h^{\min \left(\eta_{f}, \eta_{L}, \eta_{\alpha}\right)} & \longrightarrow 0, \\
\sqrt{\bar{k} h^{p}} \alpha\left(n / \bar{k} \mid x_{0}\right) h^{\min \left(\eta_{M}, \eta_{\beta}\right)} & \longrightarrow 0, \\
\sqrt{k h^{p}} \alpha\left(n / k \mid x_{0}\right) & \longrightarrow \infty, \\
\sqrt{\bar{k} h^{p}} \alpha\left(n / \bar{k} \mid x_{0}\right) \beta\left(n / \bar{k} \mid x_{0}\right) & \longrightarrow \mu_{1}\left(x_{0}\right) \in \mathbb{R}_{+} \\
\sqrt{\bar{k} h^{p}} \alpha^{2}\left(n / \bar{k} \mid x_{0}\right) & \longrightarrow \mu_{2}\left(x_{0}\right) \in \mathbb{R}_{+}
\end{aligned}
$$


and for some $q>1$ and $0<\eta<\min \left(\eta_{F_{1}}, \ldots, \eta_{F_{d}}\right)$

$$
n \sqrt{\frac{h^{p}}{k}} \max \left(\sqrt{\frac{\mid \log c^{q}}{n c^{p}}}, c^{\eta}\right) \longrightarrow 0
$$

Then we have

$$
\begin{gathered}
\sqrt{k h^{p}}\left\{\bar{L}_{k, \bar{k}}\left(y \mid x_{0}\right)-L\left(y \mid x_{0}\right)-\alpha\left(\frac{n}{k} \mid x_{0}\right) \beta\left(\frac{n}{k} \mid x_{0}\right) N\left(y \mid x_{0}\right) c\left(r ; \rho\left(x_{0}\right)+\rho^{\prime}\left(x_{0}\right)\right)\right. \\
\left.-\frac{\alpha^{2}\left(n / k \mid x_{0}\right)}{2} \frac{M^{2}\left(y \mid x_{0}\right)}{L\left(y \mid x_{0}\right)} d\left(r, \xi ; \rho\left(x_{0}\right)\right)\right\} \stackrel{d}{\longrightarrow} \frac{1}{r-1} \int_{1}^{r} \frac{B(a y)}{a} d a
\end{gathered}
$$

in $D\left([\varepsilon, T]^{d}\right)$, for every $\varepsilon>0$ and $T>\varepsilon$, where $B$ is defined in Theorem 2.1.

Note that this bias-corrected estimator $\bar{L}_{k, \bar{k}}\left(\cdot \mid x_{0}\right)$ has the same asymptotic variance as the uncorrected estimator $\breve{L}_{k}\left(\cdot \mid x_{0}\right)$ (see Theorem 2.2). Also, the conditions on $k, \bar{k}, h$ and $c$ appearing in Theorem 2.5 can be satisfied with $\mu_{1}\left(x_{0}\right)=\mu_{2}\left(x_{0}\right)=0$ if one chooses, up to multiplicative constants, $k=n^{\alpha_{1}}$, $\bar{k}=n^{\alpha_{2}}, h=n^{-\Delta_{1}}$ and $c=n^{-\Delta_{2}}$, where the parameters satisfy the following bounds

$$
\begin{aligned}
& \max \left\{\frac{-\rho\left(x_{0}\right)\left(1+\frac{2 \min \left\{\eta_{f}, \eta_{L}, \eta_{\alpha}\right\}}{p}\right)}{-\rho\left(x_{0}\right)\left(1+\frac{2 \min \left\{\eta_{f}, \eta_{L}, \eta_{\alpha}\right\}}{p}\right)+\frac{\min \left\{\eta_{f}, \eta_{L}, \eta_{\alpha}\right\}}{p}}, \frac{-2 \rho\left(x_{0}\right)}{1-2 \rho\left(x_{0}\right)}, \frac{1-2 \rho\left(x_{0}\right)}{2-2 \rho\left(x_{0}\right)},\right. \\
& \left.1-\frac{\eta}{\left(1-\rho\left(x_{0}\right)\right)(2 \eta+p)}\right\}<\alpha_{1}<1, \\
& \alpha_{1}<\alpha_{2}<\min \left\{\left[\alpha_{1}\left(1-2 \rho\left(x_{0}\right)\right)+2 \rho\left(x_{0}\right)\right]\left(1+\frac{2 \min \left\{\eta_{f}, \eta_{L}, \eta_{\alpha}\right\}}{p}\right)\right. \text {, } \\
& \frac{\left[\alpha_{1}\left(1-2 \rho\left(x_{0}\right)\right)+2 \rho\left(x_{0}\right)\right]\left(1+\frac{2 \min \left\{\eta_{M}, \eta_{\beta}\right\}}{p}\right)-2 \rho\left(x_{0}\right)}{1-2 \rho\left(x_{0}\right)}, \\
& \left.\frac{\alpha_{1}\left(1-2 \rho\left(x_{0}\right)\right)-2 \rho^{\prime}\left(x_{0}\right)}{1-2\left(\rho\left(x_{0}\right)+\rho^{\prime}\left(x_{0}\right)\right)}, \frac{\alpha_{1}\left(1-2 \rho\left(x_{0}\right)\right)-2 \rho\left(x_{0}\right)}{1-4 \rho\left(x_{0}\right)}, 1\right\}, \\
& \max \left\{\frac{\alpha_{2}}{p+2 \min \left\{\eta_{f}, \eta_{L}, \eta_{\alpha}\right\}}, \frac{\alpha_{2}\left(1-2 \rho\left(x_{0}\right)\right)+2 \rho\left(x_{0}\right)}{p+2 \min \left\{\eta_{M}, \eta_{\beta}\right\}},\right. \\
& \frac{\alpha_{2}\left(1-4 \rho\left(x_{0}\right)\right)+4 \rho\left(x_{0}\right)}{p}, \frac{\alpha_{2}+2\left(1-\alpha_{2}\right)\left(\rho\left(x_{0}\right)+\rho^{\prime}\left(x_{0}\right)\right)}{p}, \frac{1-\alpha_{1}}{p}, \\
& \left.\frac{2(\eta+p)-\alpha_{1}(2 \eta+p)}{p(2 \eta+p)}\right\}<\Delta_{1}<\frac{\alpha_{1}\left(1-2 \rho\left(x_{0}\right)\right)+2 \rho\left(x_{0}\right)}{p}, \\
& \frac{2-\alpha_{1}-\Delta_{1} p}{2 \eta}<\Delta_{2}<\Delta_{1}-\frac{1-\alpha_{1}}{p} \text {. }
\end{aligned}
$$

According to the above, one can start with choosing $\alpha_{1}$ within its bounds. Then, given $\alpha_{1}$, one can choose $\alpha_{2}$, and so on. It is thus possible to find sequences $k$, $\bar{k}, h$ and $c$ satisfying the conditions of the theorem. 


\section{Simulation study}

Our aim in this section is to illustrate the bias-correcting effect in the estimation of $L\left(\cdot \mid x_{0}\right)$. We focus on dimensions $d=2$ and $p=1$. We consider the two models studied in Escobar-Bach et al. (2018b), which both satisfy our third order condition, together with Assumptions $(\mathcal{D}),(\mathcal{L}),(\mathcal{A}),(\mathcal{B}),(\mathcal{M})$ and $\left(\mathcal{F}_{m}\right)$. In particular, these models are the following:

- Model 1: The bivariate Student distribution with density function

$$
f_{\left(Y^{(1)}, Y^{(2)}\right)}\left(y_{1}, y_{2}\right)=\frac{\sqrt{1-\theta^{2}}}{2 \pi}\left(1+\frac{y_{1}^{2}-2 \theta y_{1} y_{2}+y_{2}^{2}}{\nu}\right)^{-\frac{\nu+2}{2}}, \quad\left(y_{1}, y_{2}\right) \in \mathbb{R}^{2},
$$

where $\theta$ is the Pearson correlation coefficient. The stable tail dependence function can be described as

$$
L\left(y_{1}, y_{2} \mid \theta\right)=y_{1} F_{\nu+1}\left(\frac{\left(y_{1} / y_{2}\right)^{1 / \nu}-\theta}{\sqrt{1-\theta^{2}}} \sqrt{\nu+1}\right)+y_{2} F_{\nu+1}\left(\frac{\left(y_{2} / y_{1}\right)^{1 / \nu}-\theta}{\sqrt{1-\theta^{2}}} \sqrt{\nu+1}\right),
$$

where $F_{\nu+1}$ is the distribution function of the univariate Student distribution with $(\nu+1)$ degrees of freedom. Also

$$
\begin{aligned}
& M\left(y_{1}, y_{2} \mid \theta\right)= C_{1}\left[y_{1}^{2 / \nu+1} F_{\nu+3}\left(\frac{\left(y_{1} / y_{2}\right)^{1 / \nu}-\theta}{\sqrt{1-\theta^{2}}} \sqrt{\nu+3}\right)\right. \\
&\left.+y_{2}^{2 / \nu+1} F_{\nu+3}\left(\frac{\left(y_{2} / y_{1}\right)^{1 / \nu}-\theta}{\sqrt{1-\theta^{2}}} \sqrt{\nu+3}\right)\right], \\
& N\left(y_{1}, y_{2} \mid \theta\right)= C_{2}\left[y_{1}^{4 / \nu+1} F_{\nu+5}\left(\frac{\left(y_{1} / y_{2}\right)^{1 / \nu}-\theta}{\sqrt{1-\theta^{2}}} \sqrt{\nu+5}\right)\right. \\
&\left.+y_{2}^{4 / \nu+1} F_{\nu+5}\left(\frac{\left(y_{2} / y_{1}\right)^{1 / \nu}-\theta}{\sqrt{1-\theta^{2}}} \sqrt{\nu+5}\right)\right], \\
& C_{1}:=-\frac{\nu^{2 / \nu+1} \pi^{1 / \nu}(\nu+1)}{2(\nu+2)}\left(\frac{\Gamma\left(\frac{\nu}{2}\right)}{\Gamma\left(\frac{\nu+1}{2}\right)}\right)^{2 / \nu}, \\
& C_{2}:= \frac{\nu^{4 / \nu+1} \pi^{2 / \nu}(\nu+1)(\nu+3)}{8(\nu+4)}\left(\frac{\Gamma\left(\frac{\nu}{2}\right)}{\Gamma\left(\frac{\nu+1}{2}\right)}\right)^{4 / \nu}, \\
& \alpha(t \mid \theta)= t^{-2 / \nu}, \\
& \beta(t \mid \theta)= t^{-2 / \nu} .
\end{aligned}
$$

We set $\theta=X$, where $X$ is uniformly distributed on $[0,1]$. In the simulations, we use $\nu=4$, which corresponds to $\rho\left(x_{0}\right)=\rho^{\prime}\left(x_{0}\right)=-1 / 2$. For this model we have $\eta_{F_{1}}=\eta_{F_{2}}=\eta_{f}=\eta_{L}=\eta_{\alpha}=\eta_{\beta}=\eta_{M}=1$ and we set $\eta=0.99$. Also, the conditions of Theorem 2.5 on $k, \bar{k}, h$ and $c$ can be satisfied by using the bounds (2.5)-(2.8). For instance, if one takes $\alpha_{1}=0.80, \alpha_{2}=0.81, \Delta_{1}=0.54$ and $\Delta_{2}=0.335$, then the theoretical convergence rate is of order $n^{-0.13}$. 
- Model 2: a particular case of the Archimax bivariate copulas introduced in Capéraà et al. (2000) and also mentioned in Fougères et al. (2015), namely:

$$
C\left(y_{1}, y_{2} \mid x\right)=\left\{1+L\left(y_{1}^{-1}-1, y_{2}^{-1}-1 \mid x\right)\right\}^{-1},
$$

where we use for $L$ the asymmetric logistic stable tail dependence function defined by

$$
L\left(y_{1}, y_{2} \mid x\right)=\left(1-t_{1}\right) y_{1}+\left(1-t_{2}\right) y_{2}+\left[\left(t_{1} y_{1}\right)^{\theta_{x}}+\left(t_{2} y_{2}\right)^{\theta_{x}}\right]^{1 / \theta_{x}}
$$

where $0 \leqslant t_{1}, t_{2} \leqslant 1$, and $\theta_{x}:=\min (1 / x, 100)$, with the covariate $X$ uniformly distributed on $[0,1]$. The marginal distributions are taken to be unit Fréchet. For this model

$$
\begin{aligned}
M\left(y_{1}, y_{2} \mid x\right)= & y_{1}^{2} \partial_{1} L\left(y_{1}, y_{2} \mid x\right)+y_{2}^{2} \partial_{2} L\left(y_{1}, y_{2} \mid x\right)-L^{2}\left(y_{1}, y_{2} \mid x\right) \\
N\left(y_{1}, y_{2} \mid x\right)= & \partial_{1} L\left(y_{1}, y_{2} \mid x\right)\left(y_{1}^{3}-2 L\left(y_{1}, y_{2} \mid x\right) y_{1}^{2}\right) \\
& +\partial_{2} L\left(y_{1}, y_{2} \mid x\right)\left(y_{2}^{3}-2 L\left(y_{1}, y_{2} \mid x\right) y_{2}^{2}\right)+\frac{1}{2} \partial_{11} L\left(y_{1}, y_{2} \mid x\right) y_{1}^{4} \\
& +\frac{1}{2} \partial_{22} L\left(y_{1}, y_{2} \mid x\right) y_{2}^{4}+\partial_{12} L\left(y_{1}, y_{2} \mid x\right) y_{1}^{2} y_{2}^{2}+L^{3}\left(y_{1}, y_{2} \mid x\right), \\
\alpha(t \mid x)= & t^{-1}, \\
\beta(t \mid x)= & t^{-1} .
\end{aligned}
$$

Hence $\rho\left(x_{0}\right)=\rho^{\prime}\left(x_{0}\right)=-1$. In the simulations, different values for the pair $\left(t_{1}, t_{2}\right)$ have been tried but the results seem to be not too much influenced by them, thus we exhibit only the results in case $\left(t_{1}, t_{2}\right)=(0.4,0.6)$ which corresponds to an asymmetric tail dependence function. For this model we have $\eta_{F_{1}}=\eta_{F_{2}}=\eta_{f}=\eta_{L}=\eta_{\alpha}=\eta_{\beta}=\eta_{M}=1$ and we set $\eta=0.99$. The bounds (2.5)-(2.8) are satisfied with, e.g., $\alpha_{1}=0.84, \alpha_{2}=0.85, \Delta_{1}=$ $0.5, \Delta_{2}=0.335$, leading to a rate of $n^{-0.17}$.

For each model, we simulate 500 samples of size 1000, and we compare three estimators of $L\left(\cdot \mid x_{0}\right)$ : the two uncorrected estimators, $\widehat{L}_{k}\left(\cdot \mid x_{0}\right)$ and its smoothed version $\breve{L}_{k}\left(\cdot \mid x_{0}\right)$, and our bias-corrected estimator $\bar{L}_{k, \bar{k}}\left(\cdot \mid x_{0}\right)$, at position $x_{0}=$ 0.3. Concerning the kernel, we always use the bi-quadratic function

$$
K(u):=\frac{15}{16}\left(1-u^{2}\right)^{2} \mathbb{1}_{\{u \in[-1,1]\}} .
$$

Each estimator requires the selection of some tuning parameters. This will be done as follows.

For the uncorrected estimator $\widehat{L}_{k}\left(\cdot \mid x_{0}\right)$ of Escobar-Bach et al. $(2018 \mathrm{~b})$, we follow their approach, i.e., we use their cross-validation criterion for both bandwidth parameters $c_{1}$ and $c_{2}$, corresponding to the marginals approximation, and for the sequence $h$, we use

$$
h=\frac{\min \left(c_{1}, c_{2}\right)}{\left|\log \left(\min \left(c_{1}, c_{2}\right)\right)\right|^{1.1}} \frac{k}{n},
$$


coming from condition (2.1), as described in their paper.

For the uncorrected smoothed estimator $\breve{L}_{k}\left(\cdot \mid x_{0}\right)$, the pair $(r, \xi)$ is selected in a data-driven way using the homogeneity of the function $L\left(y \mid x_{0}\right)$, namely, for all $y$ and $k$

$$
\left(r^{*}, \xi^{*}\right):=\underset{(r, \xi) \in R \times E}{\operatorname{argmin}} \sum_{t \in T}\left(\breve{L}_{k}\left(t y \mid x_{0}\right)-t \breve{L}_{k}\left(y \mid x_{0}\right)\right)^{2},
$$

where $R:=\{1.1,1.2, \ldots, 2\}, E:=\{1,2,3\}$ and $T:=\{1 / 3,2 / 3,1,4 / 3,5 / 3\}$. The grids of values are selected after an extensive simulation study.

For the bias-corrected estimator $\bar{L}_{k, \bar{k}}\left(\cdot \mid x_{0}\right)$, also a data-driven method has been used for all the parameters involved. More precisely, $\bar{L}_{k, \bar{k}}\left(\cdot \mid x_{0}\right)$ defined in (2.4) is based on the uncorrected smoothed estimator $\breve{L}_{k}\left(\cdot \mid x_{0}\right)$ computed with $\left(r^{*}, \xi^{*}\right)$ from which we remove the bias, based on estimates $\check{\alpha}_{\bar{k}}\left(\cdot \mid x_{0}\right)$ and $\breve{\rho}_{\bar{k}}\left(x_{0}\right)$, derived according to the following algorithm:

Step 1. Let $y^{*}=(0.5,0.5), s=0.4$ and $\bar{k}=\left\lfloor n^{0.999}\right\rfloor$. Note that this heuristic choice of $\bar{k}$ works well for a wide range of distributions, and also in practical applications;

Step 2. Note that $\check{\rho}_{\bar{k}}\left(x_{0}\right)$ is an estimate of $\rho\left(x_{0}\right)$, and as such is independent of $y$. Define $\mathcal{R}:=\left\{\left(r_{1}, r_{2}\right) \in R^{2}: r_{1} \neq r_{2}\right\}, \Xi:=\left\{\left(\xi_{1}, \xi_{2}, \xi_{3}, \xi_{4}\right) \in E^{4}: \xi_{1}=\right.$ $\left.\xi_{3}, \xi_{2}=\xi_{4}\right\}$ and denote $\check{\rho}_{\bar{k}}\left(x_{0}, y^{*}, r_{1}, r_{2}, \xi_{1}, \xi_{2}, \xi_{3}, \xi_{4}\right):=\check{\rho}_{\bar{k}}\left(x_{0}\right)$ as in (2.2) for all $\left(r_{1}, r_{2}\right) \in \mathcal{R},\left(\xi_{1}, \xi_{2}, \xi_{3}, \xi_{4}\right) \in \Xi$ and $y=y^{*}$. Then, find $\left(r_{1}^{*}, r_{2}^{*}, \xi_{1}^{*}, \xi_{2}^{*}\right)$ the values of $\left(\breve{r}_{1}, \breve{r}_{2}, \breve{\xi}_{1}, \breve{\xi}_{2}\right) \in \mathcal{R} \times E^{2}$ minimizing the criterion

$\sum_{\left(r_{1}, r_{2}, \xi_{1}, \xi_{2}\right) \in \mathcal{R} \times E^{2}}\left(\check{\rho}_{\bar{k}}\left(x_{0}, y^{*}, \check{r}_{1}, \check{r}_{2}, \check{\xi}_{1}, \check{\xi}_{2}, \check{\xi}_{1}, \check{\xi}_{2}\right)-\check{\rho}_{\bar{k}}\left(x_{0}, y^{*}, r_{1}, r_{2}, \xi_{1}, \xi_{2}, \xi_{1}, \xi_{2}\right)\right)^{2}$.

The estimate $\breve{\rho}_{\bar{k}}\left(x_{0}\right)$ in (2.4) is finally computed as $\check{\rho}_{\bar{k}}\left(x_{0}, y^{*}, r_{1}^{*}, r_{2}^{*}, \xi_{1}^{*}, \xi_{2}^{*}, \xi_{1}^{*}, \xi_{2}^{*}\right)$; Step 3. Let $\check{\alpha}_{\bar{k}}\left(\cdot, r_{3}, r_{4}, \xi_{5}, \xi_{6} \mid x_{0}\right):=\check{\alpha}_{\bar{k}}\left(\cdot \mid x_{0}\right)$ as defined in (2.3). We use the homogeneity of $M\left(\cdot \mid x_{0}\right)$ in order to select the parameters $\left(r_{3}, r_{4}, \xi_{5}, \xi_{6}\right)$. More precisely, $\check{\alpha}_{\bar{k}}\left(\cdot \mid x_{0}\right)$ in (2.4) is computed as $\check{\alpha}_{\bar{k}}\left(\cdot, r_{3}^{*}, r_{4}^{*}, \xi_{5}^{*}, \xi_{5}^{*} \mid x_{0}\right)$ where

$\left(r_{3}^{*}, r_{4}^{*}, \xi_{5}^{*}\right)$

$$
:=\underset{\left(r_{3}, r_{4}, \xi_{5}\right) \in \mathcal{R} \times E}{\operatorname{argmin}} \sum_{t \in T}\left(\check{\alpha}_{\bar{k}}\left(t y^{*}, r_{3}, r_{4}, \xi_{5}, \xi_{5} \mid x_{0}\right)-t^{1-\check{\rho}_{\bar{k}}\left(x_{0}\right)} \check{\alpha}_{\bar{k}}\left(y^{*}, r_{3}, r_{4}, \xi_{5}, \xi_{5} \mid x_{0}\right)\right)^{2} .
$$

In the latter, $\check{\rho}_{\bar{k}}\left(x_{0}\right)$ is the value obtained in Step 2.

First, in order to assess the theoretical result provided by Theorem 2.5, we show in Figure 1 the associated normal QQ-plots for the two models, three different sample sizes $n=1000,5000$ and 10000, and the theoretical choices of the parameters $k, \bar{k}, h, c$ induced by the conditions of Theorem 2.5. Here $y=$ $(0.4,0.6)$ and $x_{0}=0.3$, but similar plots can be obtained for other $y$-positions and values of the covariate $x_{0}$. From these normal QQ-plots, we can see that the linearity in the plots improves with increasing the sample size $n$ and the points are getting closer to the diagonal, though there remains some curvature in the QQ-plot for Model 2, even for $n=10000$.

Next, in order to assess the effect of replacing the unknown margins by estimators, we plot in Figure 2 the empirical quantiles of the normalized estimator 

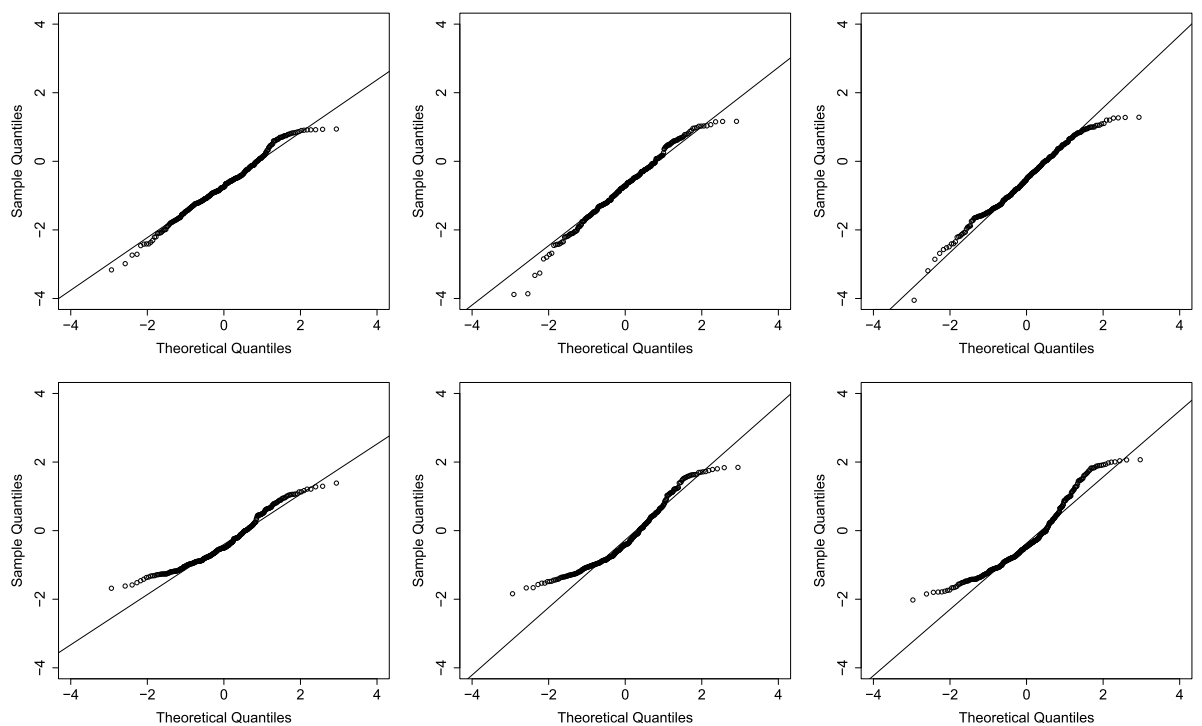

FIG 1. Normal QQ-plots of $\bar{L}_{k, \bar{k}}(0.4,0.6 \mid 0.3)$, correctly normalized, in accordance with Theorem 2.5 for Model 1 (first row) and Model 2 (second row) and three values of the sample size: $n=1000$ (first column), $n=5000$ (second column), $n=10000$ (third column).

$\widehat{L}_{k}(0.4,0.6 \mid 0.3)$ (see Theorem 2.1) with estimated margins versus those obtained with the true margins. Again the QQ-plots have been constructed with the theoretical choices of $k, h$ and $c$ and two different sample sizes: $n=1000$ (left column) and $n=5000$ (right column). As is clear from Figure 2, the similarity between the two quantiles in Model 1 is better than in Model 2, and as expected, increasing the sample size improves the results, with points getting closer to the diagonal. Again, similar results can be obtained for other $y$-positions and values of the covariate $x_{0}$.

Finally, in Figure 3, we show the sample mean (left) and the empirical mean squared error (MSE, right) of $\widehat{L}_{k}\left(y \mid x_{0}\right)$ (dotted line), $\breve{L}_{k}\left(y \mid x_{0}\right)$ (dashed line) and $\bar{L}_{k, \bar{k}}\left(y \mid x_{0}\right)$ (full line) as a function of $k$ in case of Model 1 with $x_{0}=0.3$ and four possible values of $y$, corresponding to the different rows: from the top to the bottom, $y=(0.2,0.8),(0.4,0.6),(0.6,0.4)$ and $(0.8,0.2)$, respectively. The horizontal line on the left panel represents the true value of $L\left(y \mid x_{0}\right)$. Figure 4 concerns Model 2 and the same values of $x_{0}$ and $y$. Based on these simulations, we can draw the following conclusions:

- Our estimator $\bar{L}_{k, \bar{k}}\left(y \mid x_{0}\right)$ clearly outperforms the two alternatives. In terms of bias, the sample means show very stable paths as a function of $k$, close to the true value. In terms of MSE, it is still competitive, almost always better than $\widehat{L}_{k}\left(y \mid x_{0}\right)$ and $\breve{L}_{k}\left(y \mid x_{0}\right)$, or otherwise at least similar, and again very stable as a function of $k$. Those are very nice features since in our case, the selection of $k$ is not very crucial, while it is for $\widehat{L}_{k}$ and $\breve{L}_{k}$. 

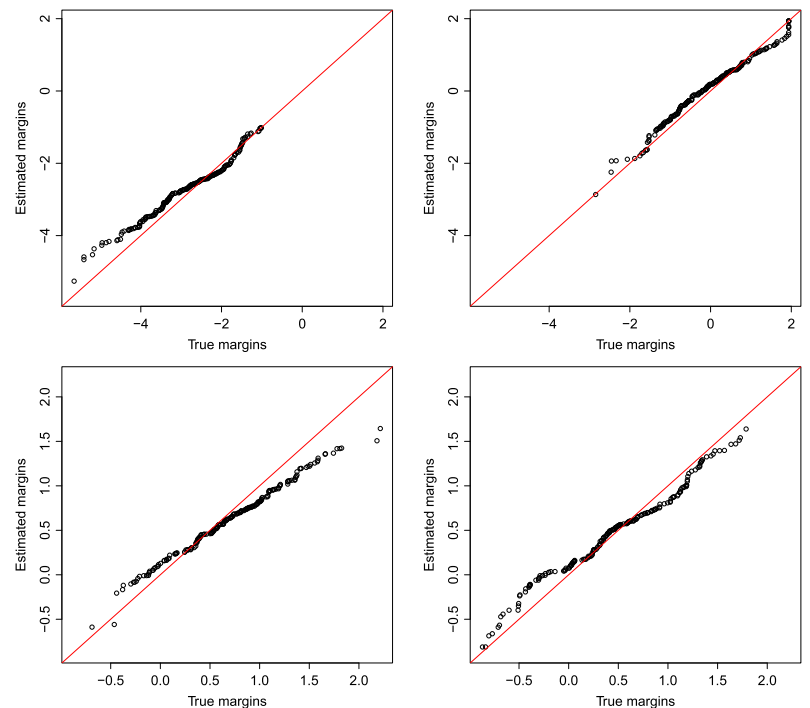

FIG 2. Empirical quantiles of the normalized estimator $\widehat{L}_{k}(0.4,0.6 \mid 0.3)$ with estimated margins versus those obtained with the true margins for Model 1 (first row) and Model 2 (second row) and two values of the sample size: $n=1000$ (left column) and $n=5000$ (right column).

- For Model 2, the estimation is more difficult for $y$ far away from the diagonal, whereas for Model 1, it does not depend on $y$.

- Different values of the covariate $x_{0}$ have been tried, but since the performance of our bias-corrected estimator $\bar{L}_{k, \bar{k}}\left(y \mid x_{0}\right)$ does not seem to depend on the position in the covariate space, the figures are not included in the paper.

\section{Application to air pollution data}

In this section, we illustrate the practical applicability of our bias-corrected estimator on a dataset of air pollution measurements. We consider the data collected by the United States Environmental Protection Agency (EPA), publicly available at https://aqsdr1.epa.gov/aqsweb/aqstmp/airdata/download_files.html. The dataset contains daily measurements of, among others, maximum temperature, ground-level ozone, carbon monoxide and particulate matter concentrations, for the period 1999 to 2013, and this for stations spread over the U.S. Monitoring levels of these pollutants is of crucial importance, as extreme temperature and high levels of pollutants like ground-level ozone and particulate matter pose a major threat to human health. We estimate the stable tail dependence function for the variables temperature and ozone concentration, conditional on time and location, where the latter is expressed by latitude and longitude. In the estimation, the covariates are standardised to the interval $[0,1]$, and the 

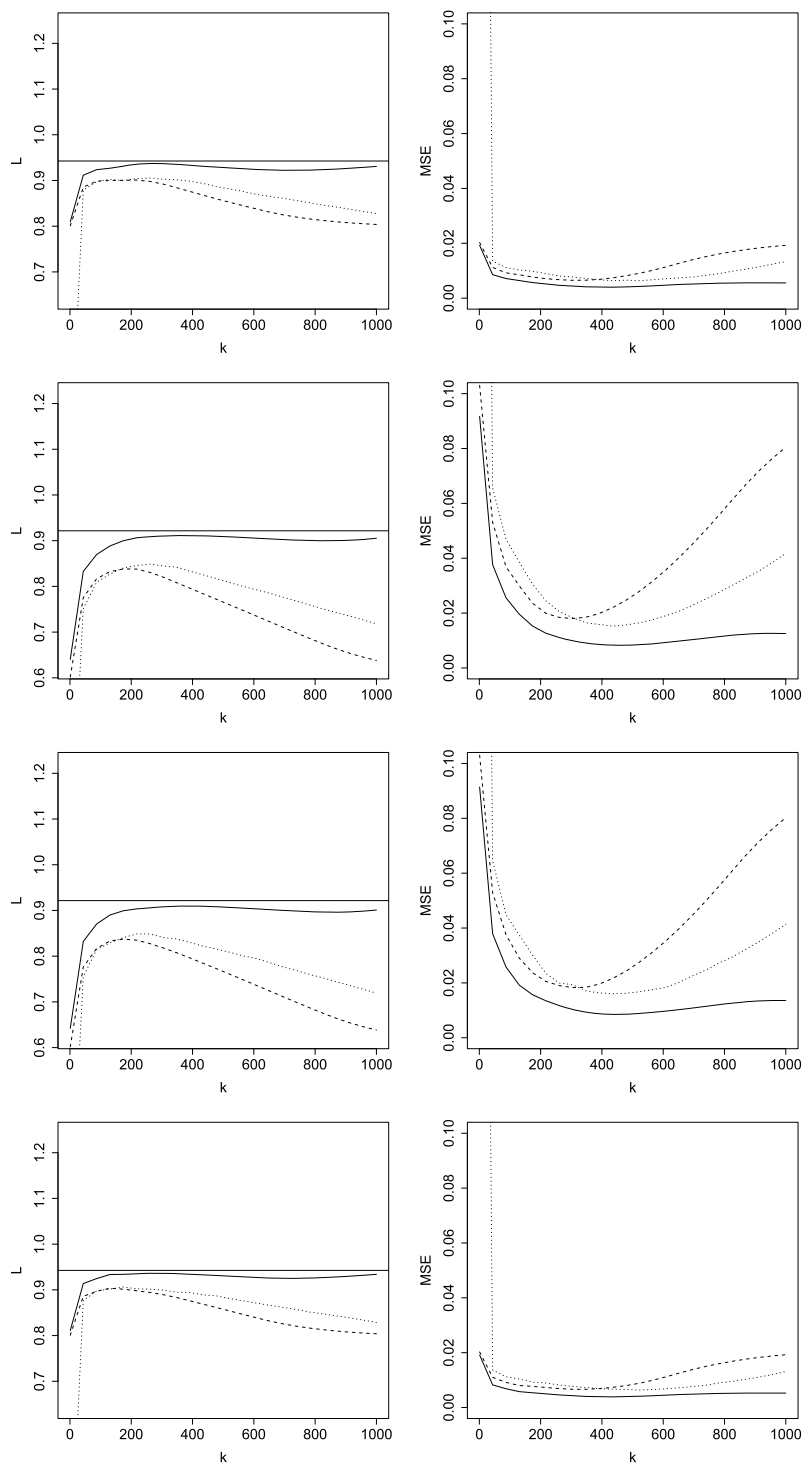

FIG 3. Model 1: Mean (left) and MSE (right) of three estimators of $L(y \mid 0.3): \widehat{L}_{k}(y \mid 0.3)$ (dotted line), $\breve{L}_{k}(y \mid 0.3)$ (dashed line), $\bar{L}_{k, \bar{k}}(y \mid 0.3)$ (full line) as a function of $k$ for different values of $y$ corresponding to each row: $y=(0.2,0.8),(0.4,0.6),(0.6,0.4),(0.8,0.2)$. The horizontal line on the left panel corresponds to the true value of $L(y \mid 0.3)$.

tuning parameters are selected with the algorithm described in Section 3. In order to keep the computational time requirements under control, the tuning parameters selected at steps 1 . to 3 . of the algorithm are computed with a random sampling of size $\lceil 0.1 n\rceil$ where $n=127328$ refers to the initial sample size. 

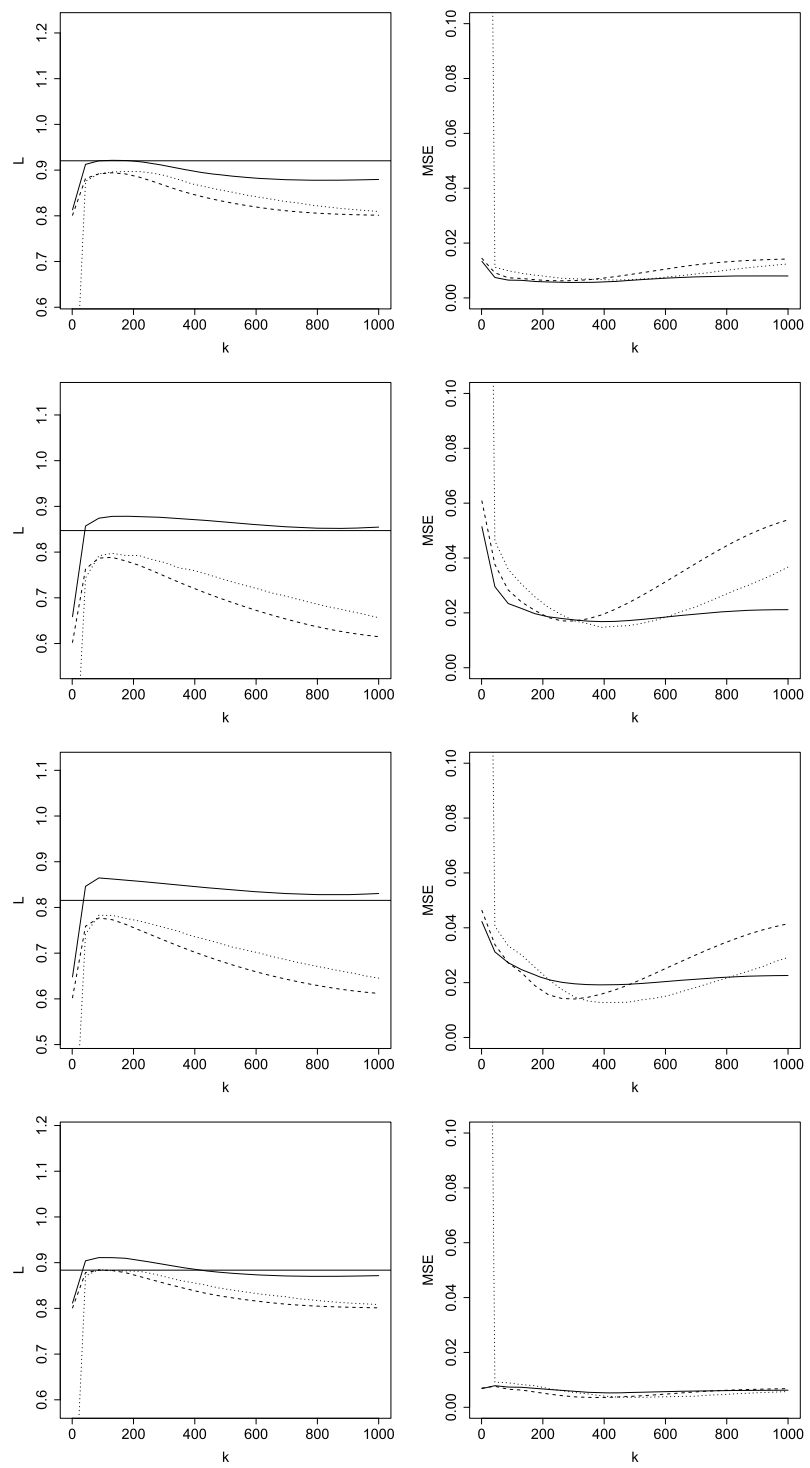

FIG 4. Model 2: Mean (left) and MSE (right) of three estimators of $L(y \mid 0.3): \hat{L}_{k}(y \mid 0.3)$ (dotted line), $\breve{L}_{k}(y \mid 0.3)$ (dashed line), $\bar{L}_{k, \bar{k}}(y \mid 0.3)$ (full line) as a function of $k$ for different values of $y$ corresponding to each row: $y=(0.2,0.8),(0.4,0.6),(0.6,0.4),(0.8,0.2)$. The horizontal line on the left panel corresponds to the true value of $L(y \mid 0.3)$.

As kernel function $K^{*}$, we use the following generalisation of the bi-quadratic kernel $K$ :

$$
K^{*}\left(x_{1}, x_{2}, x_{3}\right):=\prod_{i=1}^{3} K\left(x_{i}\right)
$$


where $x_{1}, x_{2}, x_{3}$, refer to the covariates time, latitude and longitude, respectively, in standardised form. Note that $K^{*}$ has as support the unit ball with respect to the max-norm on $\mathbb{R}^{3}$.

We report here only the results at two different time points, January 15 , 2007 and June 15, 2007, and for two locations, Fresno and Los Angeles (both in California). In Figure 5, we show the estimates median $\left\{\widetilde{L}_{k}(t, 1-t \mid x), k=\right.$ $n / 4, \cdots, n / 2\}$, with a range of $k$-values based on 25 equally spaced integers, where $\widetilde{L}_{k}$ is either $\bar{L}_{k, \bar{k}}$ (full line), $\widehat{L}_{k}$ (dotted line) or $\breve{L}_{k}$ (dashed line), for the cities Fresno (top row) and Los Angeles (bottom row) on January 15, 2007 (first column) and June 15, 2007 (second column). For both stations, the biascorrected estimate for the stable tail dependence function indicates a stronger extreme dependence between temperature and ozone concentration in winter than in summer. In winter the extreme dependence in Fresno is stronger than in Los Angeles. The results obtained with the uncorrected estimators $\breve{L}_{k}$ and $\breve{L}_{k}$ are typically similar to each other, and correspond more or less with the analysis reported in Escobar-Bach et al. (2018b). The estimate $\bar{L}_{k, \bar{k}}$ differs considerably from $\widehat{L}_{k}$ and $\breve{L}_{k}$ for Fresno, winter and Los Angeles, summer. Note that in these cases, the bias-corrected estimate tends to be higher than the uncorrected estimates, indicating a weaker extremal dependence. This was also observed in the simulation experiment, where the bias-corrected estimator tends to be larger (and closer to the true value) than the uncorrected estimators. The observed discrepancy indicates that estimation of tail dependence between temperature and ozone concentration can suffer from bias, and therefore it is recommended to use the bias-corrected estimator in order to get a better estimate of the stable tail dependence function.

\section{Proofs}

\subsection{Proof of Theorem 2.1}

We follow the lines of proof of Theorems 2.1 and 2.3 in Escobar-Bach et al. (2018b). The only difference lies in the fact that, under the third order condition, we have

$$
\begin{aligned}
& \frac{n}{k} \mathbb{P}\left(A_{n / k, y} \mid X=x_{0}-h u\right)-L\left(y \mid x_{0}-h u\right) \\
& =\alpha\left(\frac{n}{k} \mid x_{0}-h u\right) M\left(y \mid x_{0}-h u\right)+\alpha\left(\frac{n}{k} \mid x_{0}-h u\right) \beta\left(\frac{n}{k} \mid x_{0}-h u\right) N\left(y \mid x_{0}-h u\right) \\
& +\alpha\left(\frac{n}{k} \mid x_{0}-h u\right) \beta\left(\frac{n}{k} \mid x_{0}-h u\right)
\end{aligned}
$$

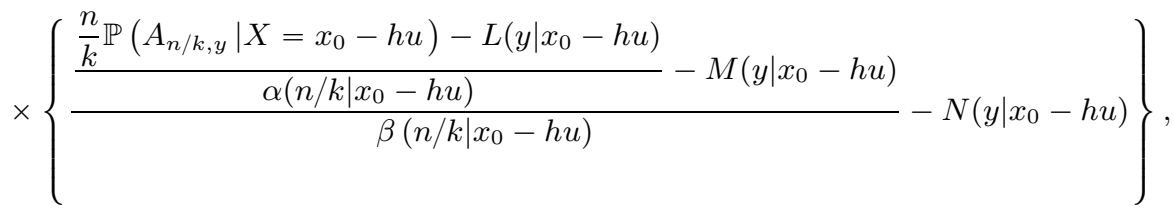



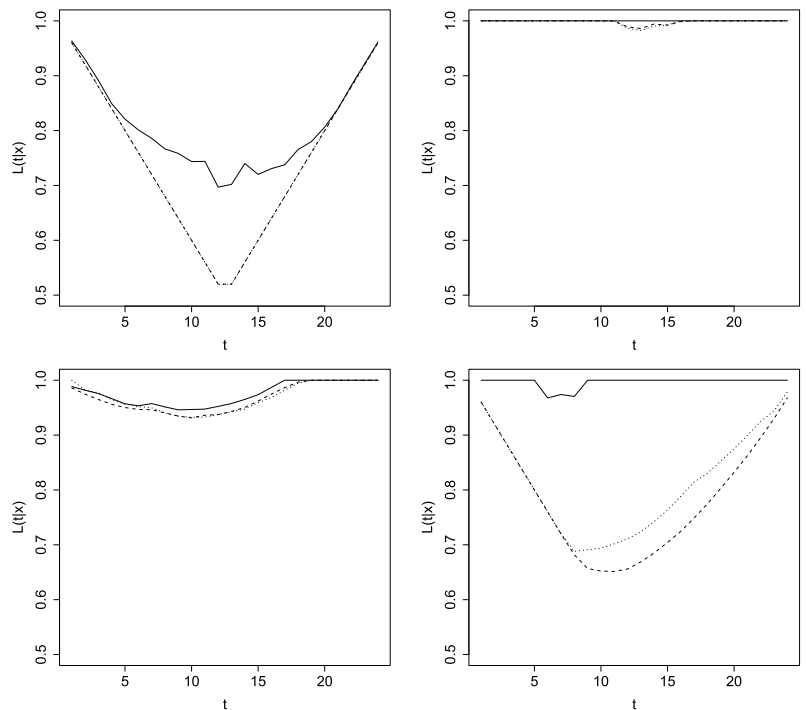

FIG 5. Air pollution data: Estimates of median $\left\{\widetilde{L}_{k}(t, 1-t \mid x), k=n / 4, \cdots n / 2\right\}$, with a range of $k$-values based on 25 equally spaced integers, for Fresno (top) and Los Angeles (bottom) on January 15, 2007 (first column) and June 15, 2007 (second column).

where

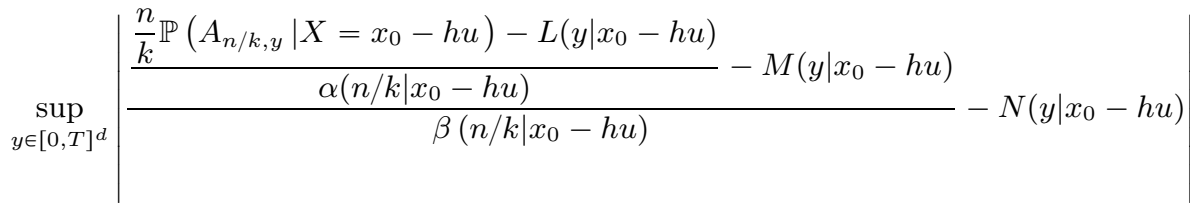

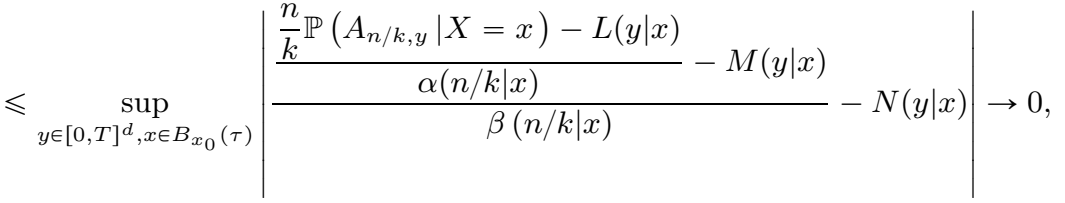

since for $n$ large enough $x_{0}-h u \in B_{x_{0}}(\tau)$. This leads to

$$
\begin{aligned}
\frac{n}{k} \mathbb{P}\left(A_{n / k, y} \mid X=x_{0}-h u\right)-L\left(y \mid x_{0}-h u\right) & \\
= & \alpha\left(\frac{n}{k} \mid x_{0}\right) M\left(y \mid x_{0}-h u\right)+\alpha\left(\frac{n}{k} \mid x_{0}\right) \beta\left(\frac{n}{k} \mid x_{0}\right)\left[N\left(y \mid x_{0}-h u\right)+o(1)\right] \\
& +\left[\alpha\left(\frac{n}{k} \mid x_{0}-h u\right)-\alpha\left(\frac{n}{k} \mid x_{0}\right)\right] M\left(y \mid x_{0}-h u\right) \\
& +\left[\alpha\left(\frac{n}{k} \mid x_{0}-h u\right) \beta\left(\frac{n}{k} \mid x_{0}-h u\right)-\alpha\left(\frac{n}{k} \mid x_{0}\right) \beta\left(\frac{n}{k} \mid x_{0}\right)\right]\left[N\left(y \mid x_{0}-h u\right)+o(1)\right] \\
=\quad & \alpha\left(\frac{n}{k} \mid x_{0}\right) M\left(y \mid x_{0}\right)+\alpha\left(\frac{n}{k} \mid x_{0}\right) \beta\left(\frac{n}{k} \mid x_{0}\right) N\left(y \mid x_{0}\right) \\
& +O\left(\alpha\left(\frac{n}{k} \mid x_{0}\right) h^{\eta_{M} \wedge \eta_{\beta}}\right)+O\left(h^{\eta_{\alpha}}\right)+o\left(\alpha\left(\frac{n}{k} \mid x_{0}\right) \beta\left(\frac{n}{k} \mid x_{0}\right)\right),
\end{aligned}
$$


where the error terms are all independent from $y$.

\subsection{Proof of Theorem 2.2}

Using Theorem 2.1, the homogeneity properties of the functions $L\left(\cdot \mid x_{0}\right), M\left(\cdot \mid x_{0}\right)$, $N\left(\cdot \mid x_{0}\right)$, and the Skorohod representation (but keeping the same notation), we have

$$
\begin{aligned}
\widehat{L}_{k, a}\left(y \mid x_{0}\right) \stackrel{d}{=} L\left(y \mid x_{0}\right) & +\alpha\left(\frac{n}{k} \mid x_{0}\right) a^{-\rho\left(x_{0}\right)} M\left(y \mid x_{0}\right) \\
& +\alpha\left(\frac{n}{k} \mid x_{0}\right) \beta\left(\frac{n}{k} \mid x_{0}\right) a^{-\rho\left(x_{0}\right)-\rho^{\prime}\left(x_{0}\right)} N\left(y \mid x_{0}\right) \\
& +\frac{1}{\sqrt{k h^{p}}} \frac{B(a y)}{a}+\frac{1}{a} o\left(\frac{1}{\sqrt{k h^{p}}}\right),
\end{aligned}
$$

where the $o$-term is almost surely and uniform in $a$ and $y$. This implies that for any $r>1$ and $\xi>0$, under the assumptions of Theorem 2.2, we have by the Skorohod representation and a straightforward application of Taylor's theorem that

$$
\begin{aligned}
\frac{\int_{1}^{r} \hat{L}_{k, a}^{\xi}\left(y \mid x_{0}\right) d a}{r-1} \stackrel{d}{=} & L^{\xi}\left(y \mid x_{0}\right)+\alpha\left(\frac{n}{k} \mid x_{0}\right) \xi L^{\xi-1}\left(y \mid x_{0}\right) M\left(y \mid x_{0}\right) c\left(r ; \rho\left(x_{0}\right)\right) \\
& +\alpha\left(\frac{n}{k} \mid x_{0}\right) \beta\left(\frac{n}{k} \mid x_{0}\right) \xi L^{\xi-1}\left(y \mid x_{0}\right) N\left(y \mid x_{0}\right) c\left(r ; \rho\left(x_{0}\right)+\rho^{\prime}\left(x_{0}\right)\right) \\
& +\alpha^{2}\left(\frac{n}{k} \mid x_{0}\right) \frac{\xi(\xi-1)}{2} L^{\xi-2}\left(y \mid x_{0}\right) M^{2}\left(y \mid x_{0}\right) c\left(r ; 2 \rho\left(x_{0}\right)\right) \\
& +\frac{1}{\sqrt{k h^{p}}} \frac{\xi}{r-1} L^{\xi-1}\left(y \mid x_{0}\right) \int_{1}^{r} \frac{B(a y)}{a} d a+o\left(\frac{1}{\sqrt{k h^{p}}}\right) .
\end{aligned}
$$

Theorem 2.2 follows then from another application of Taylor's theorem.

\subsection{Proof of Theorem 2.3}

According to Theorem 2.2, using the homogeneity properties of the functions $L\left(\cdot \mid x_{0}\right), M\left(\cdot \mid x_{0}\right), N\left(\cdot \mid x_{0}\right)$, and the Skorohod representation, we have

$$
\begin{aligned}
\left(\frac{\int_{1}^{r} \widehat{L}_{k, a}^{\xi}\left(s y \mid x_{0}\right) d a}{r-1}\right)^{1 / \xi} & \stackrel{d}{=} s L\left(y \mid x_{0}\right)+\alpha\left(\frac{n}{k} \mid x_{0}\right) s^{1-\rho\left(x_{0}\right)} M\left(y \mid x_{0}\right) c\left(r ; \rho\left(x_{0}\right)\right) \\
+ & \alpha\left(\frac{n}{k} \mid x_{0}\right) \beta\left(\frac{n}{k} \mid x_{0}\right) s^{1-\rho\left(x_{0}\right)-\rho^{\prime}\left(x_{0}\right)} N\left(y \mid x_{0}\right) c\left(r ; \rho\left(x_{0}\right)+\rho^{\prime}\left(x_{0}\right)\right) \\
& +\alpha^{2}\left(\frac{n}{k} \mid x_{0}\right) \frac{s^{1-2 \rho\left(x_{0}\right)}}{2} \frac{M^{2}\left(y \mid x_{0}\right)}{L\left(y \mid x_{0}\right)} d\left(r, \xi ; \rho\left(x_{0}\right)\right) \\
& +\frac{1}{\sqrt{k h^{p}}} \frac{1}{r-1} \int_{1}^{r} \frac{B(a s y)}{a} d a+o\left(\frac{1}{\sqrt{k h^{p}}}\right) .
\end{aligned}
$$

Several Taylor series expansions allow us to achieve the proof of Theorem 2.3. 


\subsection{Proof of Theorem 2.4}

Consider the decomposition

$$
\begin{aligned}
\check{\alpha}_{k}\left(y \mid x_{0}\right)= & \frac{\left(\frac{\int_{1}^{r_{3}} \hat{L}_{k, a}^{\xi_{5}}\left(y \mid x_{0}\right) d a}{r_{3}-1}\right)^{1 / \xi_{5}}-\left(\frac{\int_{1}^{r_{4}} \hat{L}_{k, a}^{\xi_{6}}\left(y \mid x_{0}\right) d a}{r_{4}-1}\right)^{1 / \xi_{6}}}{c\left(r_{3} ; \rho\left(x_{0}\right)\right)-c\left(r_{4} ; \rho\left(x_{0}\right)\right)} \\
& \times\left\{1+\frac{\left[c\left(r_{3} ; \rho\left(x_{0}\right)\right)-c\left(r_{3} ; \check{\rho}_{k}\left(x_{0}\right)\right)\right]-\left[c\left(r_{4} ; \rho\left(x_{0}\right)\right)-c\left(r_{4} ; \check{\rho}_{k}\left(x_{0}\right)\right)\right]}{c\left(r_{3} ; \check{\rho}_{k}\left(x_{0}\right)\right)-c\left(r_{4} ; \check{\rho}_{k}\left(x_{0}\right)\right)}\right\} \\
=: & T_{1}\left\{1+T_{2}\right\} .
\end{aligned}
$$

From Theorem 2.2

$$
\begin{aligned}
\frac{T_{1}}{\alpha\left(n / k \mid x_{0}\right) M\left(y \mid x_{0}\right)} \\
\stackrel{d}{=} \quad 1+\beta\left(\frac{n}{k} \mid x_{0}\right) \frac{N\left(y \mid x_{0}\right)}{M\left(y \mid x_{0}\right)} \frac{c\left(r_{3} ; \rho\left(x_{0}\right)+\rho^{\prime}\left(x_{0}\right)\right)-c\left(r_{4} ; \rho\left(x_{0}\right)+\rho^{\prime}\left(x_{0}\right)\right)}{c\left(r_{3} ; \rho\left(x_{0}\right)\right)-c\left(r_{4} ; \rho\left(x_{0}\right)\right)} \\
\quad+\alpha\left(\frac{n}{k} \mid x_{0}\right) \frac{M\left(y \mid x_{0}\right)}{L\left(y \mid x_{0}\right)} \frac{d\left(r_{3}, \xi_{5} ; \rho\left(x_{0}\right)\right)-d\left(r_{4}, \xi_{6} ; \rho\left(x_{0}\right)\right)}{2\left[c\left(r_{3} ; \rho\left(x_{0}\right)\right)-c\left(r_{4} ; \rho\left(x_{0}\right)\right)\right]} \\
+\quad \frac{1}{\sqrt{k h^{p}} \alpha\left(n / k \mid x_{0}\right) M\left(y \mid x_{0}\right)} \frac{\frac{1}{r_{3}-1} \int_{1}^{r_{3}} \frac{B(a y)}{a} d a-\frac{1}{r_{4}-1} \int_{1}^{r_{4}} \frac{B(a y)}{a} d a}{c\left(r_{3} ; \rho\left(x_{0}\right)\right)-c\left(r_{4} ; \rho\left(x_{0}\right)\right)} \\
+o\left(\frac{1}{\sqrt{k h^{p}} \alpha\left(n / k \mid x_{0}\right)}\right)
\end{aligned}
$$

and by a Taylor series expansion

$$
T_{2} \stackrel{d}{=} \frac{\left[c^{\prime}\left(r_{4} ; \rho\left(x_{0}\right)\right)-c^{\prime}\left(r_{3} ; \rho\left(x_{0}\right)\right)\right]\left(\breve{\rho}_{k}\left(x_{0}\right)-\rho\left(x_{0}\right)\right)}{c\left(r_{3} ; \rho\left(x_{0}\right)\right)-c\left(r_{4} ; \rho\left(x_{0}\right)\right)}(1+o(1)) .
$$

Combining these results leads to Theorem 2.4.

\subsection{Proof of Theorem 2.5}

From Theorem 2.2, we have

$$
\begin{aligned}
\bar{L}_{k, \bar{k}}\left(y \mid x_{0}\right) \stackrel{d}{=} L\left(y \mid x_{0}\right)+\left(\alpha\left(\frac{n}{k} \mid x_{0}\right) M\left(y \mid x_{0}\right)-\check{\alpha}_{\bar{k}}\left(y \mid x_{0}\right)\left(\frac{\bar{k}}{k}\right)^{\check{\rho}_{\bar{k}}\left(x_{0}\right)}\right) c\left(r ; \rho\left(x_{0}\right)\right) \\
\quad-\breve{\alpha}_{\bar{k}}\left(y \mid x_{0}\right)\left(\frac{\bar{k}}{k}\right)^{\check{\rho}_{\bar{k}}\left(x_{0}\right)}\left(c\left(r ; \check{\rho}_{\bar{k}}\left(x_{0}\right)\right)-c\left(r ; \rho\left(x_{0}\right)\right)\right) \\
\quad+\alpha\left(\frac{n}{k} \mid x_{0}\right) \beta\left(\frac{n}{k} \mid x_{0}\right) N\left(y \mid x_{0}\right) c\left(r ; \rho\left(x_{0}\right)+\rho^{\prime}\left(x_{0}\right)\right) \\
\quad+\frac{\alpha^{2}\left(n / k \mid x_{0}\right)}{2} \frac{M^{2}\left(y \mid x_{0}\right)}{L\left(y \mid x_{0}\right)} d\left(r, \xi ; \rho\left(x_{0}\right)\right)+\frac{1}{\sqrt{k h^{p}}} \frac{1}{r-1} \int_{1}^{r} \frac{B(a y)}{a} d a+o\left(\frac{1}{\sqrt{k h^{p}}}\right) .
\end{aligned}
$$

Now remark that, by the mean value theorem, for $\widetilde{\rho}_{\bar{k}}\left(x_{0}\right)$ an intermediate value between $\check{\rho}_{\bar{k}}\left(x_{0}\right)$ and $\rho\left(x_{0}\right)$

$$
\alpha\left(\frac{n}{k} \mid x_{0}\right) M\left(y \mid x_{0}\right)-\check{\alpha}_{\bar{k}}\left(y \mid x_{0}\right)\left(\frac{\bar{k}}{k}\right)^{\check{\rho}_{\bar{k}}\left(x_{0}\right)}
$$




$$
\begin{aligned}
& =\left\{\alpha\left(\frac{n}{k} \mid x_{0}\right) M\left(y \mid x_{0}\right)-\check{\alpha}_{\bar{k}}\left(y \mid x_{0}\right)\left(\frac{\bar{k}}{k}\right)^{\rho\left(x_{0}\right)}\right\} \\
& -\check{\alpha}_{\bar{k}}\left(y \mid x_{0}\right)\left(\frac{\bar{k}}{k}\right)^{\widetilde{\rho}_{\bar{k}}\left(x_{0}\right)} \log \frac{\bar{k}}{k}\left(\check{\rho}_{\bar{k}}\left(x_{0}\right)-\rho\left(x_{0}\right)\right) .
\end{aligned}
$$

Using Theorems 2.3 and 2.4, we can thus deduce that

$$
\begin{aligned}
\alpha & \left(\frac{n}{k} \mid x_{0}\right) M\left(y \mid x_{0}\right)-\check{\alpha}_{\bar{k}}\left(y \mid x_{0}\right)\left(\frac{\bar{k}}{k}\right)^{\check{\rho}_{\bar{k}}\left(x_{0}\right)} \\
\stackrel{d}{=} & \left\{\alpha\left(\frac{n}{k} \mid x_{0}\right)-\alpha\left(\frac{n}{\bar{k}} \mid x_{0}\right)\left(\frac{\bar{k}}{k}\right)^{\rho\left(x_{0}\right)}\right\} M\left(y \mid x_{0}\right) \\
& -\check{\alpha}_{\bar{k}}\left(y \mid x_{0}\right)\left(\frac{\bar{k}}{k}\right)^{\widetilde{\rho}_{\bar{k}}\left(x_{0}\right)} \log \frac{\bar{k}}{k}\left(\breve{\rho}_{\bar{k}}\left(x_{0}\right)-\rho\left(x_{0}\right)\right)+o\left(\frac{1}{\sqrt{k h^{p}}}\right) \\
\stackrel{d}{=} & \left\{\alpha\left(\frac{n}{k} \mid x_{0}\right)-\alpha\left(\frac{n}{\bar{k}} \mid x_{0}\right)\left(\frac{\bar{k}}{k}\right)^{\rho\left(x_{0}\right)}\right\} M\left(y \mid x_{0}\right)+o\left(\frac{1}{\sqrt{k h^{p}}}\right) .
\end{aligned}
$$

Recall that $\alpha\left(. \mid x_{0}\right)$ is regularly varying with index $\rho\left(x_{0}\right)<0$, which means that $\alpha\left(y \mid x_{0}\right)=y^{\rho\left(x_{0}\right)} \ell_{\alpha}\left(y \mid x_{0}\right)$ where $\ell_{\alpha}\left(\cdot \mid x_{0}\right)$ is a slowly varying function at infinity. Following the lines of proof of Theorem 2 in Beirlant et al. (2016), this implies that

$$
\begin{aligned}
& \alpha\left(\frac{n}{k} \mid x_{0}\right) M\left(y \mid x_{0}\right)-\check{\alpha}_{\bar{k}}\left(y \mid x_{0}\right)\left(\frac{\bar{k}}{\bar{k}}\right)^{\check{\rho}_{\bar{k}}\left(x_{0}\right)} \\
& \stackrel{\underline{d}}{=}\left(\frac{\bar{k}}{\bar{k}}\right)^{\rho\left(x_{0}\right)} \alpha\left(\frac{n}{\bar{k}} \mid x_{0}\right) \beta\left(\frac{n}{\bar{k}} \mid x_{0}\right)\left\{\frac{\frac{\ell_{\alpha}\left(n / k \mid x_{0}\right)}{\ell_{\alpha}\left(n / \bar{k} \mid x_{0}\right)}-1}{\beta\left(n / \bar{k} \mid x_{0}\right)}\right\} M\left(y \mid x_{0}\right)+o\left(\frac{1}{\sqrt{k h^{p}}}\right) \\
& =O\left(\left(\frac{\bar{k}}{k}\right)^{\rho\left(x_{0}\right)} \alpha\left(\frac{n}{\bar{k}} \mid x_{0}\right) \beta\left(\frac{n}{\bar{k}} \mid x_{0}\right)\right)+o\left(\frac{1}{\sqrt{k h^{p}}}\right) \\
& =o\left(\frac{1}{\sqrt{k h^{p}}}\right) .
\end{aligned}
$$

Consequently, since we have also by Theorem 2.3

$$
\begin{aligned}
\check{\alpha}_{\bar{k}}\left(y \mid x_{0}\right)\left(\frac{\bar{k}}{k}\right)^{\check{\rho}_{\bar{k}}\left(x_{0}\right)}\left(c\left(r ; \check{\rho}_{\bar{k}}\left(x_{0}\right)\right)-c\left(r ; \rho\left(x_{0}\right)\right)\right) & =O\left(\left(\frac{\bar{k}}{k}\right)^{\check{\rho}_{\bar{k}}\left(x_{0}\right)} \frac{1}{\sqrt{\bar{k} h^{p}}}\right) \\
& =o\left(\frac{1}{\sqrt{k h^{p}}}\right),
\end{aligned}
$$

and thus Theorem 2.5 follows.

\section{References}

Beirlant, J., Escobar-Bach, M., Goegebeur, Y. and Guillou, A. (2016). Bias-corrected estimation of stable tail dependence function. Journal of Multivariate Analysis, 143, 453-466. MR3431445 
Beirlant, J., Goegebeur, Y., Segers, J. and Teugels, J. (2004). Statistics of Extremes - Theory and Applications, Wiley Series in Probability and Statistics. John Wiley \& Sons, Inc., New York. MR2108013

Bücher, A., Segers, J. and Volgushev, S. (2014). When uniform weak convergence fails: empirical processes for dependence functions and residuals via epi- and hypographs. Annals of Statistics, 42, 1598-1634. MR3262462

Caeiro, F., Gomes, M.I. and Rodrigues, L.H. (2009). Reduced-bias tail index estimators under a third order framework. Communications in Statistics - Theory and Methods, 38, 1019-1040. MR2522545

Capérà̀, P., Fougères, A.L. and Genest, C. (2000). Bivariate distributions with given extreme value attractor. Journal of Multivariate Analysis, 72, 30-49. MR1747422

Drees, H. and HuAng, X. (1998). Best attainable rates of convergence for estimators of the stable tail dependence function. Journal of Multivariate Analysis, 64, 25-47. MR1619974

Escobar-Bach, M., Goegebeur, Y. and Guillou, A. (2018a). Local robust estimation of the Pickands dependence function. Annals of Statistics, 46, 2806-2843. MR3851756

Escobar-Bach, M., Goegebeur, Y. and Guillou, A. (2018b). Local estimation of the conditional stable tail dependence function. Scandinavian Journal of Statistics, 45, 590-617. MR3858948

Fils-Villetard, A., Guillou, A. and Segers, J. (2008). Projection estimators of Pickands dependence functions. Canadian Journal of Statistics, 36, 369-382. MR2456011

Fougères, A.L., De HaAn, L. and Mercadier, C. (2015). Bias correction in multivariate extremes. Annals of Statistics, 43, 903-934. MR3325714

Gardes, L. and Girard, S. (2015). Nonparametric estimation of the conditional tail copula. Journal of Multivariate Analysis, 137, 1-16. MR3332795

Giné, E. and Guillou, A. (2002). Rates of strong uniform consistency for multivariate kernel density estimators. Annales de l'Institut Henri Poincaré - Probabilités et Statistiques, 38, 907-921. MR1955344

Gomes, M.I., De HaAn, L. and Rodrigues, L.H. (2008). Tail index estimation for heavy-tailed models: accommodation of bias in weighted log-excesses. Journal of the Royal Statistical Society, Series B, 70, 31-52. MR2412630

DE HAAn, L. and FerReIrA, A. (2006). Extreme value theory - An introduction, Springer. MR2234156

HuAng, X. (1992). Statistics of bivariate extremes. PhD Thesis, Erasmus University Rotterdam, Tinbergen Institute Research series No. 22.

LEDFORD, A.W. and TAWn, J.A. (1997). Modelling dependence within joint tail regions. Journal of the Royal Statistical Society Series B, 59, 475-499. MR1440592

Veraverbeke, N., Omelka, M. and Gijbels, I. (2011). Estimation of a conditional copula and association measures. Scandinavian Journal of Statistics, 38, 766-780. MR2859749 